LE ELEZIONI IN ITALIA

di Aldo Di Virgilio

\title{
LE ELEZIONI POLITICHE DEL 24-25 FEBBRAIO 2013: GRANDE VOLATILITÀ, FINE DEL BIPOLARISMO, STALLO AL SENATO
}

Il contesto del voto: fine del governo tecnico, definizione dell'offerta, campagna elettorale

Le elezioni politiche 2013 sono state elezioni anticipate, convocate dopo 13 mesi di governo tecnocratico guidato dall'ex commissario europeo ed ex rettore dell'Università Bocconi Mario Monti. Si è trattato delle prime elezioni politiche del dopoguerra svoltesi in inverno: in tutta la storia repubblicana non si era mai votato in febbraio. Lo scioglimento anticipato del Parlamento era scaturito dalla decisione del PDL di non sostenere più il governo Monti e di defilarsi dalla "strana maggioranza" composta da PDL, PD e UDC che l'aveva sostenuto in Parlamento. Per tutto il 2012 tra i partiti della maggioranza e al loro interno non erano mancate tensioni. Era stato però dopo le elezioni comunali di maggio e soprattutto dopo le regionali siciliane di ottobre - consultazioni che avevano visto la forte affermazione del Movimento 5 Stelle (M5S) - che gli "spiriti animali" dei partiti si erano sintonizzati con preoccupazione sulle future elezioni politiche e che le posizioni si erano allontanate senza possibilità di ricomposizione. UDC e Terzo Polo erano rimasti allineati con il governo Monti, mentre i due partiti maggiori se ne erano allontanati: il PdL aveva iniziato a dipingerlo come «governo delle tasse», attribuendogli la responsabilità della mancata ripresa economica; il PD ne aveva criticato a più riprese il presunto atteggiamento antisindacale e la scarsa sensibilità nei confronti del lavoro e della crescente disoccupazione. Il punto di non ritorno fu raggiunto il 6 dicembre 2012, quando il PDL, per bocca di Angelino Alfano, comunicò l'intenzione del suo partito di 
"staccare la spina", costringendo Monti ad annunciare le dimissioni - sopraggiunte il 21 dicembre subito dopo l'approvazione della legge di stabilità - e il presidente Giorgio Napolitano a sciogliere le camere.

Nei mesi autunnali, in questo clima, la definizione delle alleanze elettorali e delle rispettive leadership entrò nel vivo. Ne scaturì un'offerta multipolare, con quattro-cinque attori rilevanti e la presenza, tra questi competitori, di new entries come Monti e Beppe Grillo.

La prima opzione a prendere forma è stata l'offerta di centro-sinistra. Tra fine novembre e inizio dicembre si svolsero le primarie di coalizione, con cui il centro-sinistra decise di selezionare il proprio candidato alla carica di primo ministro. Rispetto al $2008 \mathrm{vi}$ sono stati più elementi di novità: il fatto stesso che si siano svolte elezioni primarie; il fatto che si sia trattato di primarie di coalizione; il fatto che, in deroga allo statuto del partito, vi abbiano partecipato più esponenti del PD. Pierluigi Bersani aveva deciso già in giugno la convocazione di elezioni primarie, alle quali, secondo lo statuto, non sarebbe stato tenuto. La scelta di Bersani si giustificava per più ragioni. In primo luogo, il segretario puntava a rinnovare la propria legittimazione interna e a liberarsi della tutela politica dei capi corrente che l'avevano sostenuto nel 2009 nella sua corsa alla segreteria. In secondo luogo, intendeva sancire, attraverso lo svolgimento di primarie di coalizione, l'accordo elettorale con gli alleati. Si trattava di SEL, il partito di Nichi Vendola, e del Centro Democratico, formazione che, al seguito di Massimo Donadi e Bruno Tabacci, metteva assieme Diritti e Libertà, gruppo fuoriuscito dall'IDV contro la gestione di Di Pietro e la sua linea di avvicinamento al M5S, e una parte di Alleanza per l'Italia. Della coalizione, inoltre, faceva parte, in Alto Adige, la SVP (si veda la TAB. 1). Con la scelta di convocare le primarie, in terzo luogo, Bersani intendeva rispondere ai "rottamatori" di Matteo Renzi, il giovane sindaco di Firenze intenzionato a sfidare sul campo la "vecchia guardia".

La partecipazione di Renzi - diversamente dalle primarie di Prodi dell'ottobre 2005 che ne rappresentano l'unico vero antecedente - ha reso la contesa competitiva e l'esito incerto. Oltre che per generazione e ascendenza politica (PCI-PDS-DS-PD per Bersani, DC-PPI-Margherita-PD per Renzi), Bersani e Renzi erano diversi per orizzonte politico e strategia elettorale. Bersani puntava al PD come perno di una coalizione di centro-sinistra costruita assieme a SEL che, dopo il voto, si alleasse con il centro di Pier Ferdinando Casini. Renzi sembrava orientato a rilanciare l'idea del PD come partito "a vocazione maggioritaria" con cui Walter Veltroni nel 2008 aveva attestato il neonato partito a quota $33 \%$, risultato rimasto ineguagliato. Il sindaco rifiutava quindi qualsiasi alleanza con i centristi, proponendo inoltre che il partito facesse propri alcuni aspetti salienti dell'azione di governo di Monti. La competizione si giocò dapprima sul terreno delle procedure: Renzi era favorevole al turno unico e, soprattutto, a criteri permissivi di partecipazione al voto. Bersani impose il doppio turno ed eventuale ballottaggio a due e criteri di elettorato attivo più escludenti, che prevedevano una doppia registrazione $\mathrm{e}$ il divieto di partecipazione al secondo turno per elettori che non avessero preso parte al primo turno. Al conflitto sulle procedure seguì una campagna elettorale senza sconti, alla quale parteciparono cinque candidati. A Bersani e Renzi si aggiunsero il leader di SEL e governatore della regione Puglia Nichi Vendola, il consigliere regionale del PD veneto 
Laura Puppato e l'assessore al bilancio del comune di Milano nonché politico di lungo corso (DC-UDC-PPI-Margherita-API-CD) Bruno Tabacci.

TAB. 1 - L'offerta elettorale 2013 e il confronto con il 2008.

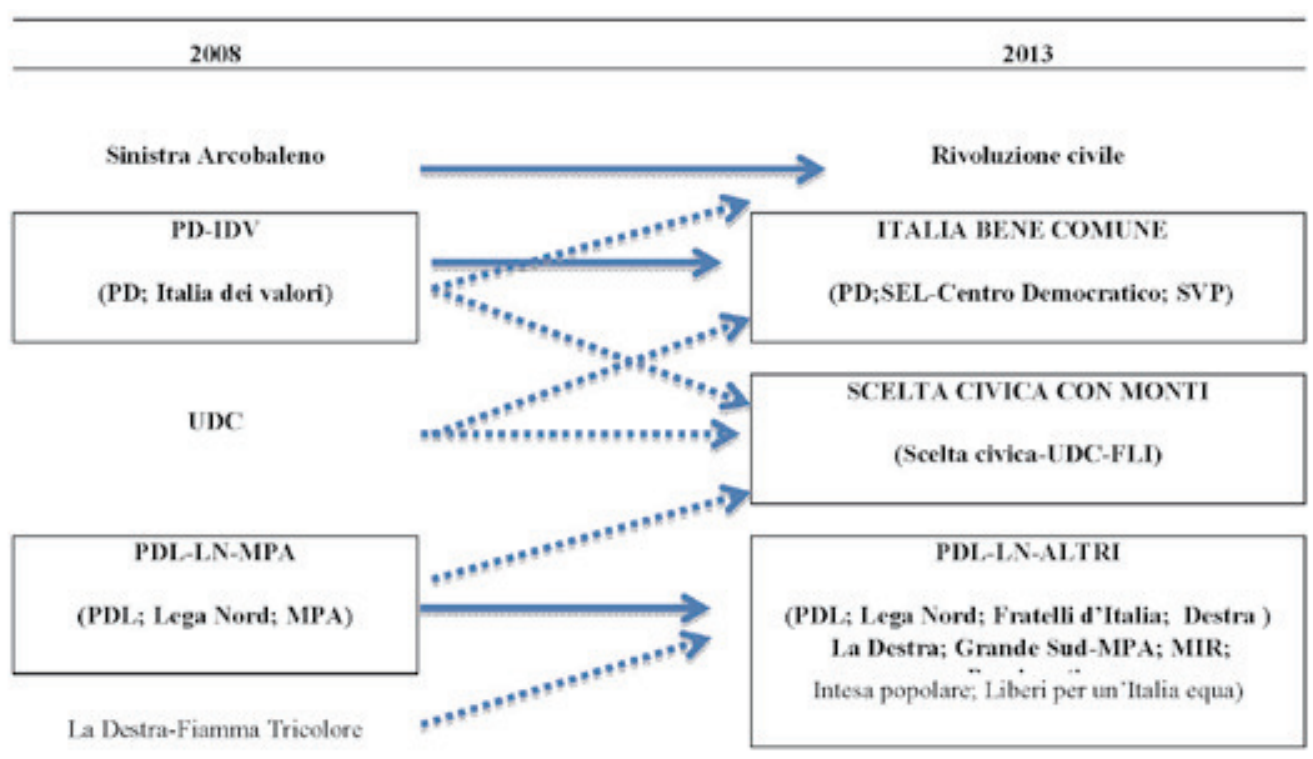

Movimento 5 Stelle (M5S)

Le ТАвB. 2 e 3 riportano i risultati dei due turni di voti che incoronarono Bersani candidato primo ministro del centro-sinistra. Al primo turno Renzi ebbe il suo punto di forza nelle regioni rosse, dove sopravanzò Bersani di quasi tre punti percentuali, e il suo punto più debole nelle regioni del Sud. Al secondo turno Bersani sconfisse Renzi con il $60 \%$ dei voti, anche grazie al sostegno dei tre candidati sconfitti al primo turno.

Dalle primarie il PD trasse indubbi vantaggi in termini di attenzione mediatica, ricavi finanziari (per prendere parte alla competizione gli oltre tre milioni di partecipanti versarono un contributo di almeno 2 euro), ritrovata legittimazione derivante dal bagno elettorale. Tutto ciò si tradusse in un balzo di circa cinque punti nel termometro delle intenzioni di voto. Combattute con asprezza, anche per la forte avversione nutrita dall'apparato e da molti dirigenti nazionali e locali nei confronti di Renzi, le primarie tuttavia prosciugarono le energie nervose del PD, aprendo ferite per sanare le quali lo scioglimento anticipato delle camere non lasciò sufficiente tempo. Inoltre, e non meno importante, con le primarie il PD scartò il candidato ritenuto più attrezzato per vincere le elezioni vere. I dati di sondaggio raccolti alla vigilia del primo turno delle primarie accreditavano un centro-sinistra guidato da Bersani del 35\% delle intenzioni di voto e un centro-sinistra guidato da Renzi del 44\%. Con Renzi in pista, inoltre, Berlusconi probabilmente avrebbe rinunciato a correre per una sesta volta, com'è invece avvenuto. 
TAB. 2 - Elezioni primarie per il candidato primo ministro della coalizione Italia bene comune (primo turno, 25 novembre 2012; valori \%).

\begin{tabular}{|c|c|c|c|c|c|c|c|}
\hline Regione & $\begin{array}{c}\text { Pier Luigi } \\
\text { Bersani }\end{array}$ & $\begin{array}{c}\text { Matteo } \\
\text { Renzi }\end{array}$ & $\begin{array}{c}\text { Nichi } \\
\text { Vendola }\end{array}$ & $\begin{array}{c}\text { Laura } \\
\text { Puppato }\end{array}$ & $\begin{array}{c}\text { Bruno } \\
\text { Tabacci }\end{array}$ & Totale & Voti validi \\
\hline Valle d'Aosta & 40,5 & 38,1 & 15,5 & 4,9 & 1,1 & 100 & 3.537 \\
\hline Piemonte & 41,7 & 38,6 & 16,0 & 2,9 & 0,8 & 100 & 177.702 \\
\hline Lombardia & 44,0 & 36,9 & 14,3 & 3,5 & 1,2 & 100 & 439.020 \\
\hline Trentino-Alto Adige & 42,9 & 35,7 & 15,4 & 5,1 & 0,8 & 100 & 26.355 \\
\hline Veneto & 41,2 & 36,4 & 11,8 & 9,9 & 0,7 & 100 & 189.337 \\
\hline Friuli-Venezia Giulia & 43,7 & 36,4 & 14,1 & 4,8 & 0,9 & 100 & 50.890 \\
\hline Liguria & 50,1 & 32,1 & 14,5 & 2,7 & 0,7 & 100 & 85.813 \\
\hline \multirow[t]{2}{*}{ Nord } & 43,5 & 36,6 & 14,2 & 4,7 & 1,0 & 100 & 972.654 \\
\hline & $(423.346)$ & $(356.335)$ & $(137.674)$ & $(45.740)$ & (9.559) & & \\
\hline Emilia Romagna & 49,0 & 38,3 & 9,8 & 2,4 & 0,6 & 100 & 439.949 \\
\hline Toscana & 35,4 & 52,1 & 10,2 & 1,8 & 0,4 & 100 & 431.573 \\
\hline Umbria & 41,8 & 45,5 & 10,9 & 1,3 & 0,5 & 100 & 77.530 \\
\hline Marche & 41,1 & 42,1 & 14,2 & 1,8 & 0,8 & 100 & 92.185 \\
\hline \multirow[t]{2}{*}{ Zona rossa } & 42,1 & 44,9 & 10,4 & 2,0 & 0,5 & 100 & 1.041 .236 \\
\hline & $(438.518)$ & $(467.417)$ & $(108.692)$ & $(21.021)$ & $(5.588)$ & & \\
\hline
\end{tabular}

\begin{tabular}{|c|c|c|c|c|c|c|c|}
\hline Lazio & 47,0 & 28,3 & 21,6 & 2,3 & 0,8 & 100 & 300.026 \\
\hline Abruzzo & 45,0 & 33,5 & 18,5 & 1,5 & 1,5 & 100 & 63.393 \\
\hline Molise & 41,7 & 31,5 & 25,3 & 0,9 & 0,6 & 100 & 11.447 \\
\hline Campania & 49,9 & 25,5 & 18,5 & 0,9 & 5,2 & 100 & 222.281 \\
\hline Puglia & 39,3 & 20,1 & 37,4 & 1,0 & 2,2 & 100 & 155.357 \\
\hline Basilicata & 56,4 & 21,4 & 17,2 & 0,7 & 4,3 & 100 & 45.168 \\
\hline Calabria & 55,0 & 22,5 & 16,5 & 0,7 & 5,3 & 100 & 104.977 \\
\hline Sicilia & 52,1 & 29,8 & 15,5 & 1,3 & 1,4 & 100 & 146.691 \\
\hline Sardegna & 53,1 & 23,3 & 20,3 & 2,2 & 1,1 & 100 & 76.163 \\
\hline \multirow[t]{2}{*}{ Sud } & 48,7 & 26,0 & 21,6 & 1,4 & 2,2 & 100 & 1.121 .940 \\
\hline & $(546.374)$ & $(292.242)$ & $(241.892)$ & $(16.217)$ & (25.215) & & \\
\hline Estero & 42,5 & 26,9 & 25,8 & 4,2 & 0,7 & 100 & 15.562 \\
\hline Totale & 44,8 & 35,5 & 15,6 & 2,7 & 1,4 & 100 & 3.154 .955 \\
\hline
\end{tabular}

Fonte: www.partitodemocratico.it

Qualche giorno dopo il successo di Bersani alle primarie, la partita delle alleanze elettorali conobbe, in rapida sequenza, tre passaggi decisivi: ritorno sulla scena di Berlusconi; dimissioni del governo Monti e scioglimento anticipato delle camere; decisione di Monti di "salire" in politica.

La configurazione della coalizione di centro-destra ebbe una gestazione difficile, connessa al ballon d'essai sul ritorno in campo di Berlusconi. Il Cavaliere aveva fatto balenare per la prima volta in giugno, davanti ai giovani del PdL, la possibilità di ricandidarsi, ma la conferma della sua decisione arrivò soltanto il 5 dicembre, al termine del 
susseguirsi di una ridda di scenari alternativi. Ai primi di ottobre Berlusconi si disse pronto a fare un passo indietro se ciò fosse servito a "unire il centro-destra" e lanciò il nome di Monti come possibile "federatore dei moderati". A fine ottobre, d'intesa con Alfano, convocò elezioni primarie per la scelta del candidato premier del centro-destra, da tenersi in dicembre sul modello della consultazione già organizzata dal centro-sinistra.

ТАВ. 3 - Elezioni primarie per il candidato primo ministro della coalizione Italia bene comune (secondo turno, 2 dicembre 2012; valori \%).

\begin{tabular}{|c|c|c|c|c|}
\hline Regione & Pier Luigi Bersani & Matteo Renzi & Totale & Voti validi \\
\hline Valle d'Aosta & 57,8 & 42,2 & 100 & 3.171 \\
\hline Piemonte & 57,9 & 42,1 & 100 & 158.907 \\
\hline Lombardia & 60,5 & 39,5 & 100 & 397.659 \\
\hline Trentino-Alto Adige & 59,7 & 40,3 & 100 & 24.082 \\
\hline Veneto & 60,7 & 39,3 & 100 & 169.944 \\
\hline Friuli-Venezia Giulia & 60,8 & 39,2 & 100 & 45.285 \\
\hline Liguria & 65,5 & 34,5 & 100 & 77.688 \\
\hline \multirow[t]{2}{*}{ Nord } & 60,3 & 39,6 & 100 & 876.736 \\
\hline & $(528.846)$ & $(346.890)$ & & \\
\hline Emilia Romagna & 60,8 & 39,2 & 100 & 406.102 \\
\hline Toscana & 45,1 & 54,9 & 100 & 399.140 \\
\hline Umbria & 51,8 & 48,2 & 100 & 70.674 \\
\hline Marche & 54,5 & 45,5 & 100 & 82.545 \\
\hline \multirow[t]{2}{*}{ Zona rossa } & 53,1 & 46,9 & 100 & 958.461 \\
\hline & $(508.560)$ & $(449.901)$ & & \\
\hline Lazio & 67,8 & 32,2 & 100 & 264.119 \\
\hline Abruzzo & 62,4 & 37,6 & 100 & 54.608 \\
\hline Molise & 63,5 & 36,5 & 100 & 9.760 \\
\hline Campania & 69,4 & 30,6 & 100 & 182.203 \\
\hline Puglia & 71,4 & 28,6 & 100 & 122.609 \\
\hline Basilicata & 71,7 & 28,3 & 100 & 37.938 \\
\hline Calabria & 74,4 & 25,6 & 100 & 93.160 \\
\hline Sicilia & 73,5 & 26,5 & 100 & 124.854 \\
\hline Sardegna & 66,5 & 33,5 & 100 & 65.839 \\
\hline \multirow[t]{2}{*}{ Sud } & 69,2 & 30,8 & 100 & 955.090 \\
\hline & $(661.259)$ & $(293.831)$ & & \\
\hline Estero & 64,4 & 35,6 & 100 & 12.095 \\
\hline Totale & 60,9 & 39,1 & 100 & 2.802.382 \\
\hline
\end{tabular}

Fonte: www.partitodemocratico.it

A inizio novembre il Cavaliere tornò però sui suoi passi e, nonostante candidature già annunciate e campagna elettorale già avviata, disdisse le primarie. Con un PdL sempre più diviso tra orientamenti anti-Europa e anti-Monti (Crosetto e Meloni) e posizioni più moderate (Frattini e Alemanno), queste giravolte servirono a Berlusconi per tenere insieme il partito e convincere i molti esponenti, ormai scettici sulla possibilità di recuperare i consensi perduti, 
di continuare a rappresentare l'unica carta spendibile. Con ciò, Berlusconi riuscì in un'operazione sulla carta quasi impossibile: far sembrare normale il suo ritorno sulla scena, in vista di una campagna "in rimonta" simile a quella del 2006 che, grazie a una piattaforma anti-tasse, anti-Germania e anti-euro, potesse assicurargli influenza e potere di veto per il dopo-elezioni. A questo scopo Berlusconi riuscì anzitutto a ricomporre l'alleanza con la Lega - la quale ritenne ragionevole scambiare il sostegno al PDL per le elezioni politiche con l'intesa sul nome di Maroni per le elezioni regionali lombarde - e chiamò a raccolta una serie di liste che resero per la prima volta la coalizione di centro-destra assai più frammentata del centro-sinistra (otto liste contro quattro: si veda ancora la TAB. 1). Tra queste liste si segnalava Fratelli d'Italia, sigla composta di fuoriusciti del PDL (La Russa, Meloni, Crosetto), che con il PDL erano però coalizzati. Un'altra peculiarità del centro-destra 2013 era infine la seguente: Berlusconi guidava lo schieramento come "capo della coalizione" ma non come candidato premier, che il centro-destra annunciò di voler scegliere "dopo il voto", contravvenendo con ciò a un suo tradizionale punto fermo.

La terza opzione elettorale, attorno alla quale si creò un clima di grande attesa mediatica, derivò dalla decisione di Monti di «salire in politica». Monti prese la decisione dopo molte incertezze, nelle settimane comprese tra l'annuncio delle dimissioni del proprio governo ( 7 dicembre, due giorni dopo la dichiarazione di ricandidatura di Berlusconi) e la loro formalizzazione (22 dicembre, il giorno seguente l'approvazione della legge di stabilità). Monti tenne a battesimo una propria lista (Scelta civica con Monti) e decise di correre alla testa di uno schieramento ispirato all'Agenda Monti, ossia alla prosecuzione dell'azione politica del suo governo. L'annuncio ufficiale arrivò il 23 dicembre, a due giorni dalle dimissioni del governo, e ricevette il plauso dell'Osservatore romano. Con Monti si schierarono UDC, FLI, l'associazione di Luca Cordero di Montezemolo Italia futura e il ministro Riccardi. La compagine decise di presentarsi come lista unica al Senato e come coalizione di tre liste alla Camera.

Il quadro dell'offerta multipolare si completò con la selezione dei candidati del M5S con elezioni primarie via web. Le cosiddette "parlamentarie" furono poco partecipate (poco più di 20 mila partecipanti sugli oltre 30 mila aventi diritto) e molto discusse, dentro e fuori il movimento di Grillo, soprattutto per i criteri dell'elettorato passivo (la possibilità di presentarsi alle primarie, e quindi di entrare nelle liste per le politiche, era limitata a esponenti del movimento che fossero già stati candidati in liste del M5S in elezioni locali senza risultare eletti).

Il 29 dicembre, infine, formalizzò la propria candidatura a premier il procuratore di Palermo Antonio Ingroia alla guida di Rivoluzione civile, lista-cartello di estrema sinistra che comprendeva partiti e movimenti che nel 2008 avevano costituito Sinistra arcobaleno (Rifondazione comunista meno SEL, Comunisti italiani, Verdi) più la parte dell'IDV rimasta con Di Pietro.

Oltre ai cinque principali competitori ai quali si è fatto cenno, e che compaiono nella $\mathrm{T}_{\mathrm{AB}}$. 1, sulle schede erano presenti i simboli di numerose liste minori, tra le quali le più votate, come si può leggere nelle tabelle in Appendice, sono state Fare per fermare il declino in Italia, il Partito comunista dei lavoratori (PCdL), Forza Nuova e Lista Amni- 
stia Giustizia e Libertà, etichetta utilizzata dai radicali per legare la loro presenza al tema della condizione carceraria e del (mal)funzionamento della giustizia in Italia, più Die Freiheitlichen, che ha raccolto 42 mila voti al Senato e 48 mila alla Camera, ossia quasi 1' $8 \%$ dei voti nella sola provincia in cui era presente (Bolzano). Il numero totale di liste concorrenti è stato così più alto rispetto alle due precedenti elezioni svoltesi con la legge Calderoli. È salito a 517 per la Camera (contro 464 del 2006 e 407 del 2008) ed a 363 per il Senato (contro 265 nel 2008 e 353 nel 2008): in media 20 liste, di cui 12 appartenenti a coalizioni e 8 non coalizzate (si veda la ТАВ. 4). Il numero medio di liste in lizza presenta una non trascurabile variazione territoriale: al Sud le schede sono risultate più affollate di simboli, anche all'interno delle coalizioni; il primato della frammentazione in entrata spetta a Campania 1 con 26 liste e Puglia e Lazio 1 con 25 per la Camera ed a Campania con 26 liste e Lombardia con 24 al Senato. Rispetto al 2008 è cresciuto anche il numero complessivo di candidature, senza però raggiungere i valori del 2006, contesa bipolare in cui si confrontarono due coalizioni catch-all (FIG. 1).

Alla campagna elettorale è mancato un denominatore comune. Ciascuna parte ha teso a enfatizzare le proprie priorità, identificando su questa base i principali avversari. Dopo aver vinto le primarie di centro-sinistra, Bersani ha attinto a piene mani alla tradizione PCI-PDS-DS e ha lasciato che su aspetti cruciali quali fiscalità, lavoro e welfare il programma fosse dettato dall'asse Fassina-Camusso-Vendola. Ha così sbilanciato a sinistra la coalizione, lasciando intendere che il vantaggio di cui il centro-sinistra era accreditato dai sondaggi fosse sufficiente per assicurare alla coalizione una maggioranza anche al Senato, nella peggiore delle ipotesi in alleanza con la coalizione Monti.

L'offerta Monti ha fatto fatica ad "arrivare" agli elettori, sia per l'inesperienza del primo ministro uscente, non sempre a suo agio nella campagna elettorale, sia per alcune ambiguità di fondo. Scelta civica, infatti, era alleata col partito di centro di Casini, ma ha rifiutato di presentarsi come partito di centro. Il partito di Monti ha invece scommesso su nuove dimensioni di competizione, in primo luogo la dimensione pro/anti Europa, ma non sempre è riuscito a comunicare con chiarezza queste diversa prospettiva.

Berlusconi ha invece impostato la campagna sull'irrilevanza dello spread e sulle congiure anti-italiane ordite in sede internazionale, elementi sui quali gli antagonisti non l'hanno chiamato ad argomentare, e, soprattutto, sul tema della restituzione e abolizione dell'Imu (Imposta municipale unica). Quest'ultimo obiettivo è stato presentato con chiarezza come provvedimento emblematico del programma anti-tasse del centro-destra. Altrettanto chiara è stata la campagna del M5S di Grillo, fondata sull'elementare ma efficace slogan "tutti a casa" e sull'interazione, tra la rete, che il Movimento ha eletto a propria "sede", la piazza (compresa, in chiusura di campagna, un'affollatissima Piazza San Giovanni a Roma, storico luogo-simbolo delle campagne elettorali del centro-sinistra, che se l'è lasciata inspiegabilmente soffiare) e la televisione, dove Grillo e i candidati a cinque stelle si sono rifiutati di andare riuscendo però a essere ugualmente e costantemente presenti per l'attenzione in diretta che alcune reti televisive hanno dedicato al Movimento. 
TAB. 4 - Elezioni politiche 2008. Numero di liste per circoscrizione e area geopolitica.

\begin{tabular}{|c|c|c|c|c|c|c|c|}
\hline & \multicolumn{3}{|c|}{ Camera } & & \multicolumn{3}{|c|}{ Senato } \\
\hline & $\begin{array}{l}\text { Liste in } \\
\text { coalizioni }\end{array}$ & $\begin{array}{l}\text { Liste non } \\
\text { coalizzate }\end{array}$ & Totale & & $\begin{array}{l}\text { Liste in } \\
\text { coalizioni }\end{array}$ & $\begin{array}{l}\text { Liste non } \\
\text { coalizzate }\end{array}$ & Totale \\
\hline Piemonte 1 & 12 & 6 & 18 & Piemonte & 9 & 7 & 16 \\
\hline Piemonte 2 & 11 & 5 & 16 & & & & \\
\hline Lombardia 1 & 12 & 8 & 20 & Lombardia & 11 & 13 & 24 \\
\hline Lombardia 2 & 11 & 5 & 16 & & & & \\
\hline Lombardia 3 & 10 & 4 & 14 & & & & \\
\hline TAA & 9 & 5 & 14 & & & & \\
\hline Veneto 1 & 12 & 11 & 23 & Veneto & 9 & 14 & 23 \\
\hline Veneto 2 & 11 & 7 & 18 & & & & \\
\hline FVG & 12 & 4 & 16 & FVG & 9 & 14 & 23 \\
\hline Liguria & 10 & 4 & 14 & Liguria & 7 & 5 & 12 \\
\hline Nord (tot.) & 110 & 59 & 169 & Nord (tot.) & 45 & 53 & 98 \\
\hline Nord (media) & 11,0 & 5,9 & 16,9 & Nord (media) & 9,0 & 10,6 & 19,6 \\
\hline Emilia Romagna & 13 & 10 & 23 & $\begin{array}{l}\text { Emilia } \\
\text { Romagna }\end{array}$ & 8 & 10 & 18 \\
\hline Toscana & 11 & 8 & 19 & Toscana & 8 & 8 & 16 \\
\hline Umbria & 13 & 3 & 16 & Umbria & 8 & 5 & 13 \\
\hline Marche & 11 & 7 & 18 & Marche & 8 & 7 & 15 \\
\hline Centro (tot.) & 48 & 28 & 76 & Centro (tot.) & 32 & 30 & 62 \\
\hline Centro (media) & 12,0 & 7,0 & 19,0 & Centro (media) & 8,0 & 7,5 & 15,5 \\
\hline Lazio 1 & 13 & 12 & 25 & Lazio & 13 & 15 & 28 \\
\hline Lazio 2 & 12 & 9 & 21 & & & & \\
\hline Abruzzo & 12 & 7 & 19 & Abruzzo & 9 & 9 & 18 \\
\hline Molise & 13 & 8 & 21 & Molise & 11 & 12 & 23 \\
\hline Campania 1 & 15 & 11 & 26 & Campania & 13 & 13 & 26 \\
\hline Campania 2 & 14 & 10 & 24 & & & & \\
\hline Puglia & 14 & 11 & 25 & Puglia & 11 & 12 & 23 \\
\hline Basilicata & 14 & 10 & 24 & Basilicata & 10 & 10 & 20 \\
\hline Calabria & 13 & 11 & 24 & Calabria & 11 & 10 & 21 \\
\hline Sicilia 1 & 12 & 6 & 18 & Sicilia & 13 & 9 & 22 \\
\hline Sicilia 2 & 12 & 10 & 22 & & & & \\
\hline Sardegna & 13 & 10 & 23 & Sardegna & 11 & 11 & 22 \\
\hline Sud (tot.) & 157 & 115 & 272 & Sud (tot.) & 102 & 101 & 203 \\
\hline Sud (media) & 13,1 & 9,6 & 22,7 & Sud (media) & 11,3 & 11,2 & 22,6 \\
\hline Italia (tot.) & 315 & 202 & 517 & Italia (tot.) & 179 & 184 & 363 \\
\hline Italia (media) & 12,1 & 7,8 & 19,9 & Italia (media) & 9,9 & 10,2 & 20,2 \\
\hline
\end{tabular}


FIG. 1 - Numero di candidature alle elezioni politiche 2001-2013 per Camera e Senato.

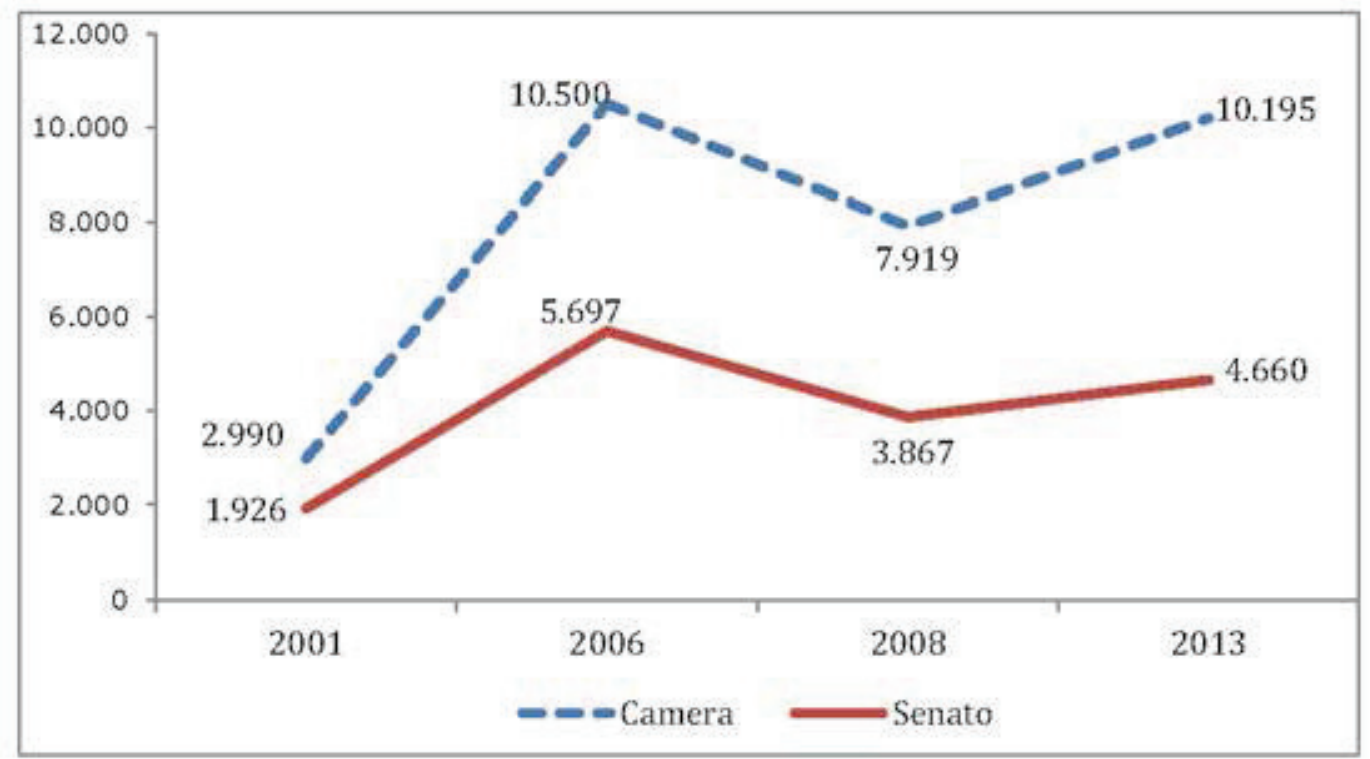

Partecipazione in calo, ma non troppo

La percentuale di votanti è stata del $75 \%$ sia alla Camera sia al Senato (TAB. 5). Si tratta di 5 punti in meno rispetto al 2008. Per la prima volta in elezioni politiche la partecipazione è scesa così sotto 1' $80 \%$ degli aventi diritto, anche se, su scala comparata, l'Italia mantiene ugualmente un tasso di partecipazione al voto tra i più alti. Considerando le schede bianche e le schede nulle, l'area del "non voto" ha superato il $27 \%$ degli iscritti. In considerazione di quanto era avvenuto nelle elezioni locali e regionali del 2012, gli osservatori avevano previsto un calo maggiore. Ė probabile che la presenza del M5S abbia catalizzato una parte di astensionismo potenziale, contribuendo a contenere la crescita del non voto.

La distribuzione geografica di questa diminuita mobilitazione rispecchia la tradizione (ТАB. 6). In Sicilia, Campania Calabria e Puglia il calo di partecipazione è stato largamente superiore alla media nazionale (come, peraltro, in Lombardia 2 e Piemonte 2). Rispetto al 2008, i livelli più bassi di astensionismo aggiuntivo si sono registrati in Molise, Piemonte 1, Valle d'Aosta e Veneto 2. 
TAв. 5 - Elezioni politiche 2013. Elettori, votanti, voti non validi, voti validi per Camera e Senato (escluse ripartizioni estero).

\begin{tabular}{lcccccc}
\hline & \multicolumn{3}{c}{ Camera } & \multicolumn{4}{c}{ Senato } \\
\cline { 2 - 7 } & N. & $\begin{array}{c}\text { \% su } \\
\text { elettori }\end{array}$ & $\begin{array}{c}\text { \% sui } \\
\text { votanti }\end{array}$ & N. & \% su elettori & \% sui votanti \\
\hline & & & & & & \\
Elettori & 47.005 .431 & & & 43.071 .530 & & \\
Votanti & 35.348 .710 & 75,2 & & 32.397 .344 & 75,2 & \\
Voti validi & 34.074 .960 & 72,5 & 96,4 & 31.228 .822 & 72,5 & 96,4 \\
Voti non validi & 1.273 .750 & & 3,6 & 1.168 .522 & & 3,6 \\
\hline
\end{tabular}

TAB. 6 - Elezioni politiche 2013. Partecipazione al voto per circoscrizione e differenze rispetto al 2008 .

\begin{tabular}{|c|c|c|c|c|c|c|c|c|c|}
\hline & \multicolumn{4}{|c|}{ Camera } & & \multicolumn{4}{|c|}{ Senato } \\
\hline & Elettori & Votanti & $\%$ & $\begin{array}{c}\Delta \\
13-08\end{array}$ & & Elettori & Votanti & $\%$ & $\begin{array}{c}\Delta \\
13-08\end{array}$ \\
\hline Valle d'Aosta & 100.277 & 77.169 & 77,0 & $-2,2$ & Valle d'Aosta & 93.040 & 71.719 & 77,1 & $-2,4$ \\
\hline Piemonte 1 & 1.771 .727 & 1.389 .249 & 78,4 & $-1,7$ & Piemonte & 3.202 .113 & 2.469 .470 & 77,1 & $-3,6$ \\
\hline Piemonte 2 & 1.667 .470 & 1.267 .959 & 76,0 & $-5,4$ & & & & & \\
\hline Lombardia 1 & 2.997 .661 & 2.376 .777 & 79,3 & $-4,3$ & Lombardia & 6.906 .677 & 5.500 .703 & 79,6 & $-5,1$ \\
\hline Lombardia 2 & 3.276 .254 & 2.619 .106 & 79,9 & $-5,9$ & & & & & \\
\hline Lombardia 3 & 1.179 .406 & 938.046 & 79,5 & $-5,0$ & & & & & \\
\hline TAA & 777.135 & 629.748 & 81,0 & $-3,3$ & TAA & 707.666 & 575.275 & 81,3 & $-3,1$ \\
\hline Veneto 1 & 2.228 .577 & 1.843 .145 & 82,7 & $-3,2$ & Veneto & 3.438 .790 & 2.806 .644 & 81,6 & $-3,0$ \\
\hline Veneto 2 & 1.488 .510 & 1.194 .009 & 80,2 & $-2,8$ & & & & & \\
\hline FVG & 964.0451 & 744.206 & 77,2 & $-3,6$ & FVG & 900.778 & 694.698 & 77,1 & $-3,6$ \\
\hline Liguria & 1.274 .561 & 957.394 & 75,1 & $-2,9$ & Liguria & 1.193 .656 & 895.193 & 75,0 & $-3,0$ \\
\hline $\begin{array}{l}\text { Emilia } \\
\text { Romagna }\end{array}$ & 3.338 .137 & 2.740 .478 & 82,1 & $-4,1$ & $\begin{array}{l}\text { Emilia } \\
\text { Romagna }\end{array}$ & 3.120 .051 & 2.559 .716 & 82,0 & $-4,2$ \\
\hline Toscana & 2.885 .048 & 2.284 .716 & 79,2 & $-4,5$ & Toscana & 2.690 .293 & 2.125 .759 & 79,0 & $-4,5$ \\
\hline Umbria & 683.834 & 543.881 & 79,5 & $-4,7$ & Umbria & 634.867 & 502.185 & 79,1 & $-4,6$ \\
\hline Marche & 1.197 .752 & 956.257 & 79,8 & $-3,1$ & Marche & 1.108 .695 & 881.014 & 79,5 & $-3,2$ \\
\hline Lazio 1 & 3.202 .373 & 2.483 .915 & 77,6 & $-3,1$ & Lazio & 4.046 .761 & 3.153 .462 & 77,9 & $-3,2$ \\
\hline Lazio 2 & 1.227 .950 & 949.876 & 77,4 & $-5,3$ & & & & & \\
\hline Abruzzo & 1.067 .298 & 810.590 & 75,9 & $-5,1$ & Abruzzo & 978.410 & 739.145 & 75,5 & $-5,3$ \\
\hline Molise & 262.008 & 204.712 & 78,1 & $-0,5$ & Molise & 238.755 & 185.666 & 77,8 & $-0,5$ \\
\hline Campania 1 & 2.418 .415 & 1.565 .441 & 64,7 & $-8,9$ & Campania & 4.074 .374 & 2.747 .504 & 67,4 & $-8,4$ \\
\hline Campania 2 & 2.175 .256 & 1.553 .198 & 71,4 & $-7,7$ & & & & & \\
\hline Puglia & 3.297 .793 & 2.306 .638 & 69,9 & $-6,3$ & Puglia & 2.968 .488 & 2.073 .406 & 69,8 & $-6,5$ \\
\hline Basilicata & 476.020 & 330.812 & 69,5 & $-5,9$ & Basilicata & 430.066 & 297.910 & 69,3 & $-6,5$ \\
\hline Calabria & 1.580 .119 & 997.905 & 63,2 & $-8,2$ & Calabria & 1.415 .635 & 889.137 & 62,8 & $-8,4$ \\
\hline Sicilia 1 & 1.953 .081 & 1.227 .651 & 62,9 & $-11,0$ & Sicilia & 3.645 .541 & 2.355 .076 & 64,6 & $-10,1$ \\
\hline Sicilia 2 & 2.123 .209 & 1.405 .186 & 66,2 & $-9,9$ & & & & & \\
\hline Sardegna & 1.391 .515 & 950.646 & 68,3 & $-4,0$ & Sardegna & 1.276 .864 & 874.662 & 68,5 & $-4,0$ \\
\hline Italia & 47.005 .431 & 35.348 .710 & 75,2 & $-5,3$ & Italia & 43.071 .520 & 32.398 .344 & 75,2 & $-5,3$ \\
\hline
\end{tabular}


Il nuovo paesaggio politico e l'influenza delle regole di voto: fine del bipolarismo e stallo al Senato

Com'era accaduto già nel 2008, anche le elezioni politiche 2013 hanno prodotto un paesaggio elettorale e parlamentare del tutto nuovo. Ancora una volta l'esito del voto ha profondamente riplasmato interazioni e rapporti di forza tra le singole formazioni politiche e ridefinito il sistema dei partiti. Ciò rappresenta un indicatore della fragilità delle formazioni politiche e della persistente fluidità del sistema dei partiti. Come si può leggere nel riepilogo generale riportato in Appendice, dalle urne sono usciti tre risultati principali: (a) lo straordinario successo del M5S, che nella sua prova d'esordio ha ottenuto il $25 \%$ dei voti (circa quattro punti in più del risultato ottenuto da Forza Italia nel 1994); (b) il flop dell'offerta Monti, che ha superato di poco il 10\% dei voti validi ed è restato al di sotto delle attese; (c) il sostanziale e imprevisto pareggio tra Bersani e Berlusconi: le due coalizioni sono rimaste entrambe sulla soglia del $30 \%$ dei voti e il centro-sinistra ha sopravanzato il centro-destra per meno di 125 mila voti alla Camera - scenario che ricorda il risultato del 2006, quando l'Unione di Prodi vinse le elezioni con un vantaggio inferiore a 25 mila voti - e per circa 280 mila voti al Senato.

Prima di esaminare in dettaglio la distribuzione del voto a coalizioni e partiti, è però opportuno fermare l'attenzione sulla distribuzione dei seggi e sui fattori istituzionali che l'hanno determinata, ossia sul funzionamento della legge elettorale di Camera e Senato.

La TAB. 7 pone a confronto la distribuzione dei seggi nelle due camere. Dai numeri emergono due aspetti principali: (a) il multipolarismo dell'offerta ha trovato conferma nella risposta degli elettori e ciò ha comportato la fine del bipolarismo parlamentare che, in forme diverse, aveva connotato la rappresentanza parlamentare nelle cinque elezioni precedenti, dal 1994 al 2008; (b) il confronto tra Camera e Senato segnala rapporti di forza assai diversi all'interno delle due camere e, al Senato, una situazione di stallo determinata dall'assenza di maggioranza.

Questi due esiti sono dipesi dall'interazione tra la distribuzione dei voti e il meccanismo di trasformazione dei voti in seggi, diverso nelle due camere. La sintesi di TAB. 8 consente di mettere a fuoco con maggior chiarezza questa interazione. Per ciascuno dei raggruppamenti che ha ottenuto seggi - identificato dal nome del rispettivo leader - la tabella pone a confronto, per Camera e Senato, percentuale di voti e percentuale di seggi. Come si può osservare, in termini di voti le differenze tra le due camere sono piccole, con uno scarto mai superiore ai due punti (come nel caso del M5S e della coalizione Monti). Le colonne delle percentuali di seggi segnalano invece differenze molto più grandi: si coglie qui l'influenza del sistema elettorale e delle differenze esistenti in proposito tra Camera e Senato. 
TAв. 7 - Elezioni politiche 2013. Distribuzione dei seggi a liste e coalizioni (Italia metropolitana).

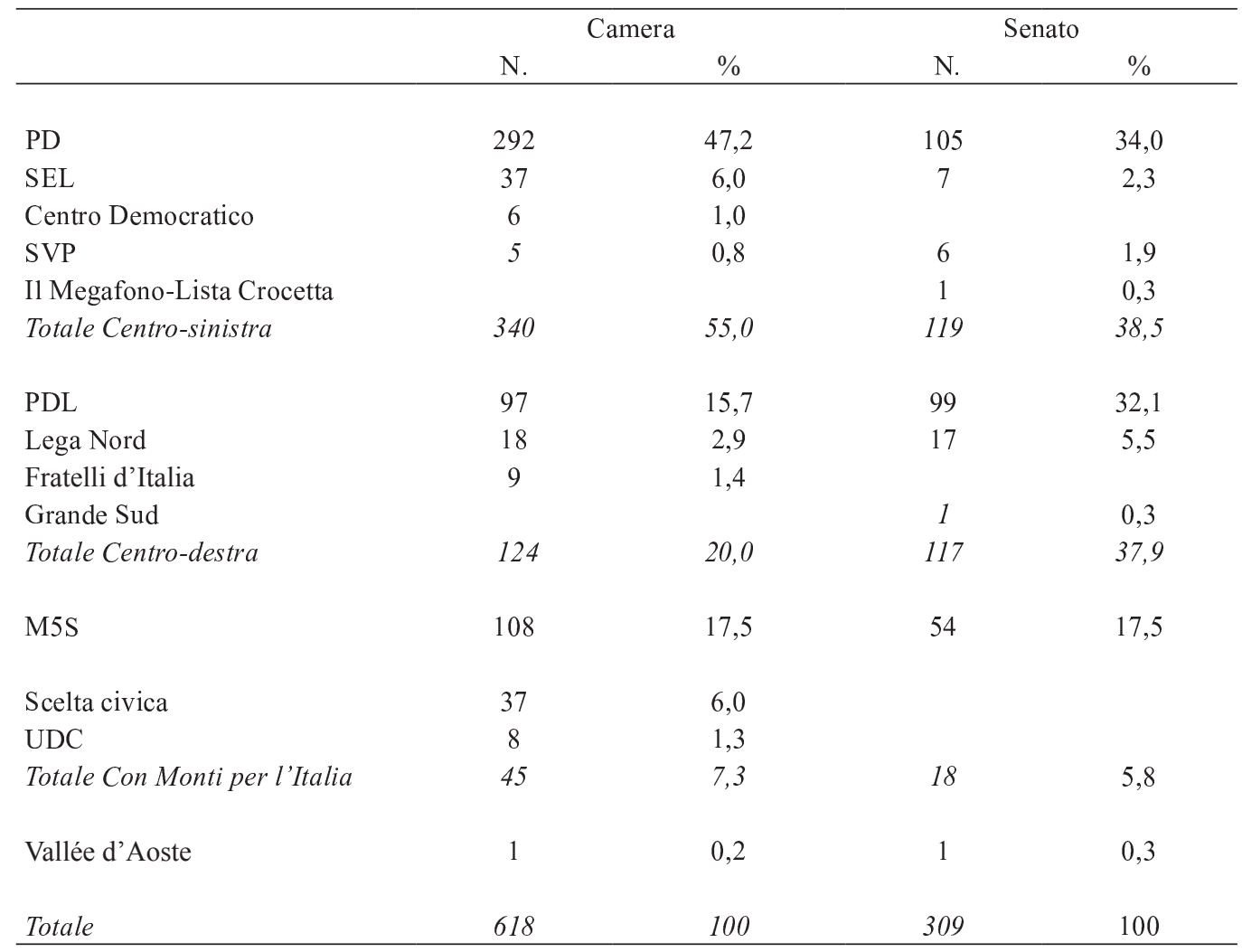

Il sistema elettorale italiano non ha ancora trovato una definizione chiara e condivisa. Alcuni l'hanno definito "sistema proporzionale con premio di maggioranza e soglie variabili", altri, con maggiore prudenza, sistema elettorale "ibrido", riconoscendo con ciò la difficoltà di ricondurlo alle categorie classificatorie prevalenti. Nel 2013 la legge Calderoli è stata utilizzata per la terza volta e ha dato luogo a effetti inediti, derivanti dal cambiamento della struttura dell'offerta elettorale, dal modo in cui la risposta fornita dagli elettori si è adattata a questo cambiamento, dalla retroazione di questo cambiamento sul meccanismo del premio di maggioranza.

Alla Camera, com'è noto, il premio di maggioranza è un premio su base nazionale (con la controversa esclusione degli elettori valdostani). Se nessun concorrente, in base alla ripartizione proporzionale dei seggi, ottiene il 55\% dei mandati (ossia 340 seggi su 617), il premio l'attribuisce alla lista o coalizione di liste con il maggior numero di voti, senza alcuna condizione. Nel 2013, con tre opzioni comprese tra il $25,6 \%$ e il $29,5 \%$ dei voti (coalizione Bersani, coalizione Berlusconi, lista Grillo), tale meccanismo ha determinato l'assegnazione di un bonus assai consistente, superiore a 25 punti di percentuale, ossia a poco meno del doppio della forza in voti del vincente (la coalizione Bersani). Ne è scaturito un picco di dis-proporzionalità, misurato nella Fıg. 2 utilizzando l'indice di Gallagher. 
TAв. 8 - Elezioni politiche 2013. Dai voti ai seggi: effetti di dis-rappresentanza per la Camera e per il Senato.

\begin{tabular}{lcccccccc}
\hline & \multicolumn{3}{c}{ Camera } & & \multicolumn{3}{c}{ Senato } \\
\cline { 2 - 3 } \cline { 7 - 8 } & \% voti & \% seggi & $\Delta$ & & \% voti & \% seggi & $\Delta$ \\
\hline Coalizione Bersani & 29,5 & 55,0 & $+25,5$ & & 31,1 & 38,5 & $+7,4$ \\
Coalizione Berlusconi & 29,1 & 20,0 & $-9,1$ & & 30,3 & 37,9 & $+7,6$ \\
Lista Grillo & 25,6 & 17,5 & $-8,1$ & & 23,6 & & 17,5 & $-6,1$ \\
Coalizione Monti & 11,0 & 7,3 & $-3,7$ & & 9,0 & 5,8 & $-3,2$ \\
\hline
\end{tabular}

Al Senato 1"“ingegnerizzazione" del premio di maggioranza ha caratteristiche diverse. Il premio, com'è noto, è attribuito in questo caso regione per regione. È quindi incapace di assicurare la costruzione di una maggioranza nell'assemblea e presenta una consistenza che dipende dal numero di seggi in palio nelle diverse circoscrizioni regionali. Nel 2013 il meccanismo del premio ha determinato al Senato un esito assai diverso da quello della Camera: non ha consentito la costruzione di una maggioranza nell'assemblea e ha prodotto una miscela di effetti distorsivi. Al Senato il livello di disproporzionalità è inferiore a quello della Camera (si veda ancora la Fig. 2), ma gli effetti del premio attribuito regione per regione sono stati incoerenti con la logica stessa del premio. Come si vede nella $\mathrm{TAB}$. 8 , il premio ha avvantaggiato più la coalizione giunta seconda per numero di voti (il centro-destra) che la coalizione con la percentuale più alta di voti complessivi (il centro-sinistra, benché per soli 8 decimi di punto). È andata così perché il centro-destra ha conquistato il premio di maggioranza nelle tre regioni più grandi: la Campania, il Lazio e, soprattutto, la Lombardia che con i suoi 49 seggi ha un peso analogo a quello dello stato dell'Ohio nella contesa presidenziale americana.

Vediamo adesso in maggior dettaglio il processo di traduzione dei voti in seggi nelle due camere e, dunque, i due sistemi elettorali in azione. La TAB. 9 presenta il processo di assegnazione dei seggi alla Camera. Nel contesto multipolare a quattro del 2013, con le tre opzioni più forti al $25-30 \%$ dei voti e l'opzione più debole attestata all' $11 \%$, la distribuzione dei seggi su base proporzionale non ha prodotto alcuna maggioranza "naturale". Poiché nessun contendente ha raggiunto quota 340, ossia il 54\% dei seggi in palio nell'Italia del premio, si è fatto così ricorso a una rideterminazione dell'assegnazione dei seggi a quozienti elettorali differenziati. La coalizione vincente - il centro-sinistra di Bersani che ha sopravanzato di 4 decimi di punto il centro-destra di Berlusconi - si è vista assegnare un premio molto grande, pari a 148 deputati (nel 2006 il premio era stato di 31 seggi, nel 2008 di 20), 127 dei quali assegnati al PD. La presenza di più minoranze ha reso il processo allocativo piuttosto articolato. 
FIG. 2 - Elezioni politiche 1976-2013: valori dell'indice di disproporzionalità di Gallagher per Camera e Senato.

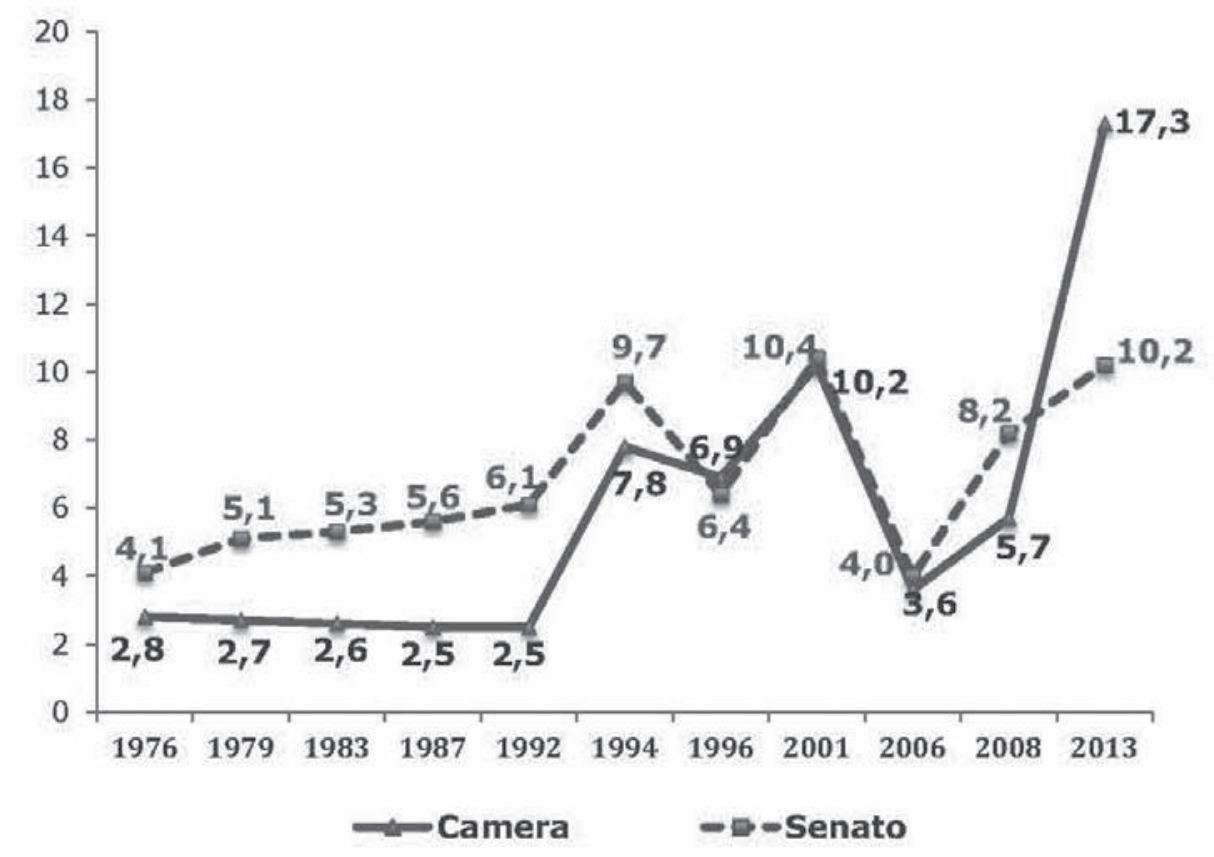

Nota: 1'Indice di Gallagher, o Least Square Index, è ottenuto elevando al quadrato e poi sommando le differenze tra le percentuali in voti e le percentuali in seggi di ogni partito. Il totale è poi diviso per due e se ne estrae la radice quadrata. L'indice fornisce una misura della disproporzionalità per elezione, piuttosto che per partito, e può variare da $0 \mathrm{a} 100$.

Come si legge nella ТАB. 10, il costo-seggi per le diverse opzioni è stato ben più diseguale di quanto non sia avvenuto nelle elezioni del 2008 e del 2006. Si tratta di un modo diverso di illustrare il fenomeno presentato sopra facendo riferimento al picco dell'indice di disproporzionalità di Gallagher (FIG. 2). Tutte le componenti della coalizione Bersani - compresi SEL, SVP e il Centro Democratico sotto soglia, che vanta il costoseggi più conveniente - hanno ottenuto un seggio con meno di 30mila voti. Al M5S ciascun seggio è invece costato oltre 80 mila voti, più di quanto non sia costato all'UDC e a Fratelli d'Italia, componenti salvate dalla clausola best loser, rispettivamente, nella coalizione Berlusconi e nella coalizione Monti. Quest'ultimo dato mostra come la coalizione possa costituire per le liste più piccole uno scudo che assicura rappresentanza a condizioni più favorevoli rispetto alle corsa delle liste non coalizzate. La TAB. 10 riporta il costo-seggi anche per le liste entrate in Senato. Anche in questo caso troviamo dati speculari a quanto sintetizzato dall'indice di Gallagher. La miscela di effetti distorsivi cui si è fatto riferimento a proposito del funzionamento della legge elettorale per la camera alta si sostanzia nel più conveniente costo-seggi spuntato dalle liste della coalizione giunta seconda, ossia PDL e Lega Nord. Questo dato, a sua volta, dipende dal fatto che il centrodestra è la coalizione vincente nella regione in cui il premio (regionale) è più consistente, ossia la Lombardia, e in due altre regioni grandi quali Lazio e Campania. 


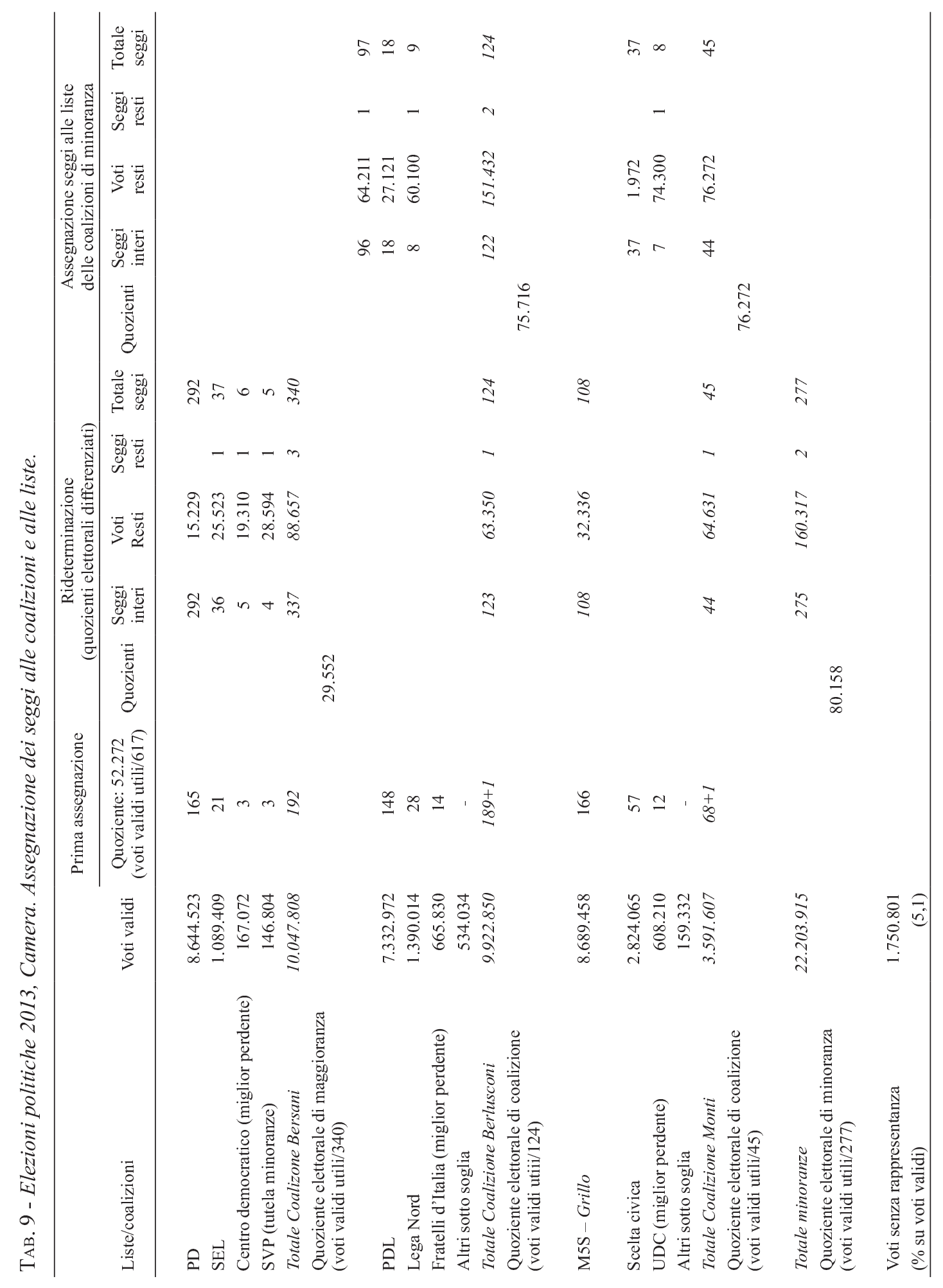


TAB. 10 - Costo-seggi per partito alla Camera e al Senato (2006-2013).

\begin{tabular}{|c|c|c|c|c|c|c|c|c|}
\hline & \multicolumn{2}{|c|}{ Costo seggi 2013} & & \multicolumn{2}{|c|}{ Costo seggi 2008} & & \multicolumn{2}{|c|}{ Costo seggi 2006} \\
\hline & Camera & Senato & & Camera & Senato & & Camera & Senato \\
\hline PD & 29.605 & 80.085 & PDL & 50.107 & 88.047 & Ulivo & 54.232 & \\
\hline SEL & 29.443 & 130.330 & Lega & 50.413 & 105.770 & DS & & 96.408 \\
\hline Centro democratico & 27.845 & & MPA & 51.311 & 177.538 & Margherita & & 93.965 \\
\hline SVP & 29.361 & & & & & $\mathrm{RC}$ & 54.377 & 93.282 \\
\hline \multirow[t]{2}{*}{$\begin{array}{l}\text { Il Megafono-Lista } \\
\text { Crocetta }\end{array}$} & & 138.581 & PD & 57.313 & 95.358 & Rosa nel pugno & 55.039 & \\
\hline & & & IDV & 56.912 & 101.008 & $\begin{array}{l}\text { Insieme per } \\
\text { l'Unione }\end{array}$ & & 219.384 \\
\hline PDL & 75.598 & 69.690 & & & & IDV & 54.816 & 246.512 \\
\hline Lega Nord & 77.223 & 78.304 & UDC & 56.953 & 632.962 & Verdi & 52.320 & \\
\hline Fratelli d' Italia & 73.981 & & & & & UDEUR & 53.409 & 158.979 \\
\hline \multirow[t]{2}{*}{ Grande Sud } & & 122.100 & & & & & & \\
\hline & & & & & & Forza Italia & & 105.150 \\
\hline \multirow[t]{2}{*}{ M5S } & 80.458 & 136.706 & & & & AN & & 103.285 \\
\hline & & & & & & UDC & & 110.069 \\
\hline Scelta civica & 76.326 & & & & & Lega Nord & & 117.841 \\
\hline UDC & 76.026 & & & & & & & \\
\hline Con Monti per l'Italia & & 155.929 & & & & & & \\
\hline
\end{tabular}

Sempre a proposito di Senato, la ТАВ. 11 consente di osservare tre aspetti importanti del processo di traduzione dei voti in seggi relativo alla camera alta. Primo: nel 2006 e 2008 la struttura bipolare aveva favorito in molte regioni l'affermazione di maggioranze "naturali" (da intendersi: superiori al 55\% dei seggi in palio nella circoscrizione) e ciò aveva limitato a poche regioni il ricorso al premio; nel 2013 la struttura multipolare della competizione ha generalizzato la necessità di far ricorso al premio di maggioranza. Secondo: se nel 2006 la somma algebrica dei premi era stata nulla e nel 2008 aveva irrobustito il vantaggio già consistente - e superiore alla maggioranza assoluta dei seggi - della coalizione vincente, nel 2013 l'effetto è stato idiosincratico. Il confronto tra l'esito della ripartizione dei seggi senza applicazione dei premi e dopo l'applicazione dei premi rende evidente, come si è già osservato, che il meccanismo del premio avvantaggia la seconda coalizione anziché la prima. Un esito incoerente con la teleologia del premio di maggioranza. Terzo: l'effetto aggregato del meccanismo, come si è detto, è l'assenza di una maggioranza nell'assemblea di Palazzo Madama. Ciò è determinato dal fatto che a contare sono le singole circoscrizioni regionali, il che assimila il sistema elettorale per il Senato al meccanismo utilizzato per designare il collegio elettorale presidenziale negli Stato Uniti. Il centro-destra si avvantaggia del meccanismo pur non avendo conseguito la maggioranza dei "voti popolari", grazie al fatto di aver conquistato il bonus in seggi nelle tre regioni in cui si assegna il maggior numero di seggi (Lombardia, Campania e Lazio), che in questa occasione si presentavano alla vigilia del voto come battleground states. 


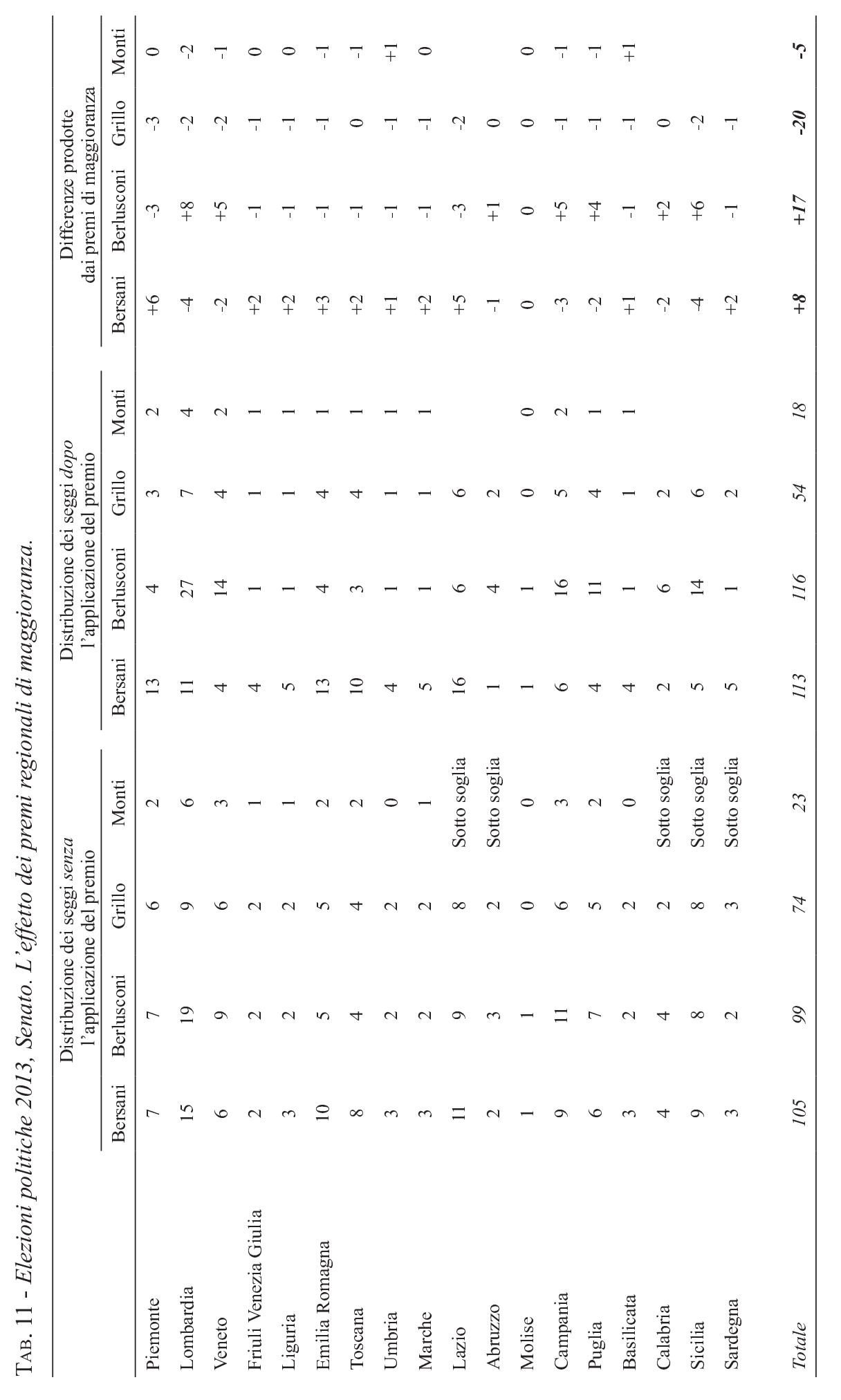




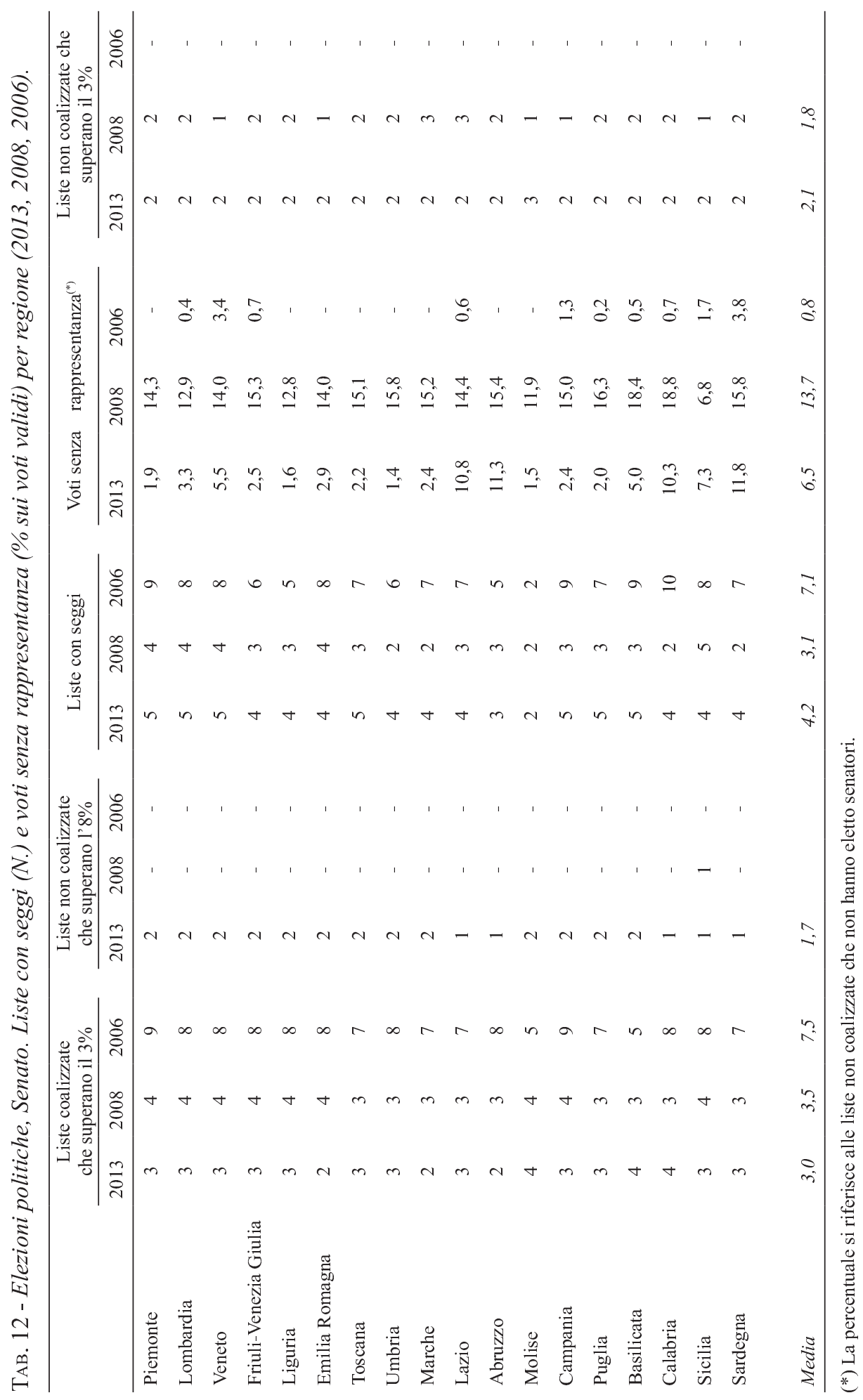


La ТАв. 12 approfondisce il confronto fra le tre elezioni per il Senato svoltesi con la legge elettorale Calderoli, presentando la struttura della competizione con riferimento alle soglie del 3\% (soglia della rappresentanza per le liste coalizzate) e dell' $8 \%$ (soglia di rappresentanza per le liste non coalizzate), al numero di liste con seggi, all'entità della quota di voti rimasti senza rappresentanza. Il dato più significativo è la presenza, nel 2013, di un numero medio di liste non coalizzate che superano 1' $8 \%$, e che accedono dunque alla rappresentanza, di poco inferiore a 2. Si tratta del M5S (in tutte le regioni) e della lista Con Monti per l'Italia (in 13 regioni su 18). Nel 2008 soltanto la lista UDC in Sicilia aveva superato tale soglia. Nel 2013, dunque, la competizione per il Senato ha visto l'irrobustimento delle terze forze. Il dato ha comportato un calo dei voti rimasti senza rappresentanza, più che dimezzato rispetto al 2008 (dal $13,7 \%$ al $6,5 \%$ ). Il numero di liste con seggi è passato da 3,1 a 4,2, senza però raggiungere il livello del 2006, quando la costruzione di coalizioni catch-all portò a più di 7 il numero medio delle liste con seggi.

$\mathrm{Nel} 2013$ è invece in lieve calo il numero di liste coalizzate che superano il 3\% dei voti. Si tratta in media di tre liste: oltre a PD e PDL, la Lega nelle regioni del Nord (ma non in Liguria) e SEL nelle regioni centro-meridionali. Sopra al 3\% troviamo inoltre il Centro democratico in Basilicata, Fratelli d'Italia in Molise e Grande Sud in Calabria. Non sempre le liste coalizzate che superano il 3\% dei voti hanno ottenuto seggi. È accaduto in due regioni piccole come Basilicata e Calabria: nella prima è restato fuori il Centro democratico col 4,4\% dei voti, nella seconda SEL col 3,96\% (mentre ha trovato il seggio Grande Sud col 3,23\%).

Riassumendo, il contesto multipolare della competizione e la presenza di tre forze di consistenza elettorale simile hanno fortemente modificato il panorama partitico. Rispetto al 2008 la concentrazione del voto (e dei seggi) sui due partiti maggiori (M5S e PD) è arretrata di circa 20 punti di percentuale (si veda la FIG. 3). Il numero di effective parties misurato nella Fig. 4 secondo l'indice di Laakso Taagepera con riferimento ai voti è tornato a crescere: il livello di frammentazione elettorale ritrova il livello del 2006 per quanto riguarda i partiti, mentre per quanto riguarda le coalizioni il quadro è simile al 1994. 
FIG. 3 - Camera dei deputati. Somma percentuale dei voti e dei seggi ai due maggiori partiti (1976-2013).

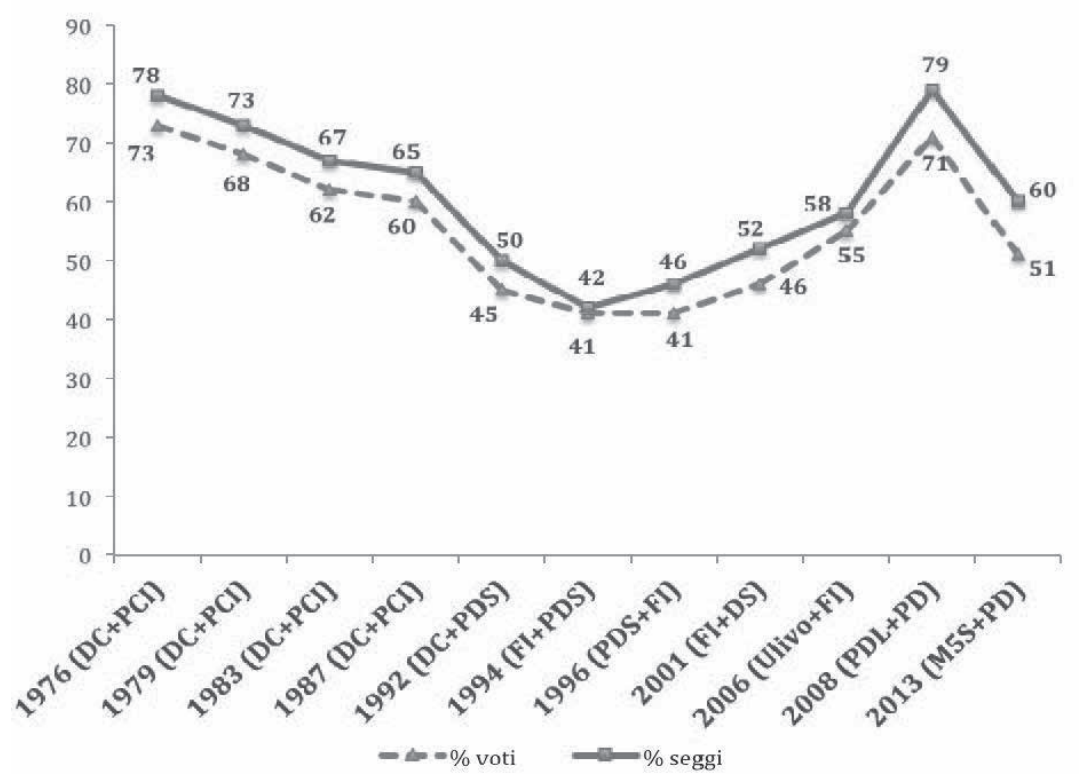

FIG. 4 - Camera dei deputati. Effective number dei partiti elettorali (1976-2013) e delle coalizioni elettorali (1994-2013) (Indice di Laakso-Taagepera).

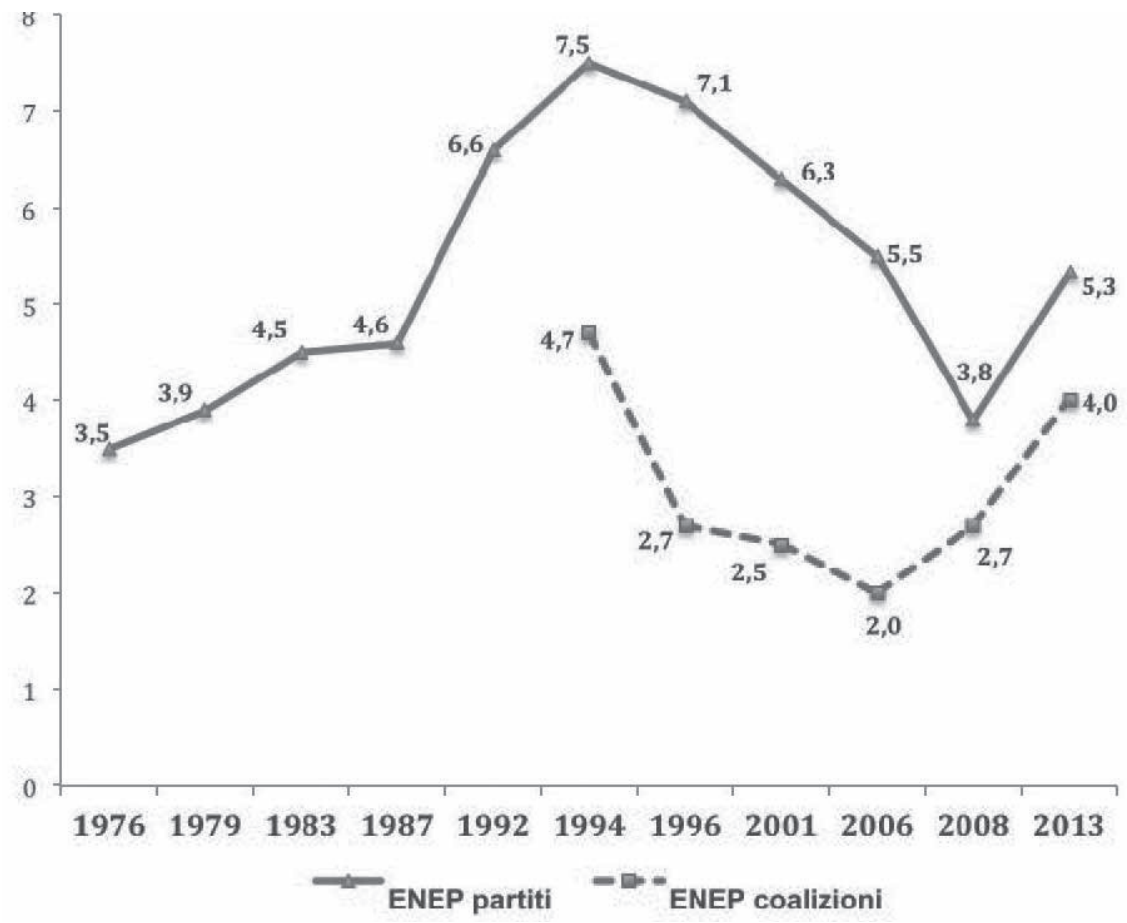

Nota: l'indice di Laaks e Taagepera è calcolato con la formula $\mathrm{N}=1 / \sum \mathrm{v}_{1}{ }^{2}\left(\mathrm{~N}=1 / \sum \mathrm{s}_{1}{ }^{2}\right)$ dove $\mathrm{v}_{1}\left(\mathrm{~s}_{1}\right)$ rappresenta la percentuale al quadrato dei voti (seggi) del partito i-esimo. È utilizzato per tenere conto al tempo stesso del numero e della forza relativa in voti (o in seggi) dei partiti presenti in un dato sistema partitico. 
Il voto a coalizioni e liste non coalizzate: successo di Grillo, flop di Monti, sostanziale pareggio tra Bersani e Berlusconi

La ТАВ. 13 reca il riepilogo del voto per le principali coalizioni e liste non coalizzate alla Camera. Quattro opzioni sono riuscite a eleggere i propri candidati, nell'ordine PD-SELCentro democratico-SVP, PDL-Lega-Fratelli d'Italia e altre liste di centro-destra, M5S e Coalizione Monti. È rimasta invece al disotto del 4\% Rivoluzione civile, la lista-cartello a trazione giudiziaria in cui assieme a Ingroia, Di Pietro e De Magistris si sono ritrovati i partiti riuniti in modo stabile, dalle elezioni europee del 2009 in avanti, sotto le bandiere della Federazione della Sinistra, ossia Verdi, Rifondazione comunista e Comunisti italiani. Questo risultato ha sancito la seconda sconfitta consecutiva della cosiddetta sinistra radicale, che, dopo l'insuccesso della Sinistra arcobaleno nel 2008, è rimasta di nuovo esclusa dalla rappresentanza parlamentare. Rivoluzione civile è risultata la quinta opzione più votata in quasi tutte le circoscrizione. Hanno fatto eccezione le tre circoscrizioni lombarde e le due venete, dove Rivoluzione civile è sopravanzata dalla lista di Oscar Giannino Fare per fermare il declino, e il Trentino Alto Adige, dove Die Freiheitlichen, grazie al 16\% ottenuto nella provincia di Bolzano, ha conquistato quasi 1' $8 \%$ su scala regionale, come si è già detto. Alla Camera è rimasto privo di rappresentanza parlamentare il $5,1 \%$ dei voti (contro il $10 \%$ del 2008).

Il verdetto delle urne ha premiato soprattutto il M5S, vero vincitore di queste elezioni. Come si può leggere nelle tabelle riportate in Appendice, il M5S è il primo partito nell'Italia del premio (in termini complessivi, ossia considerando anche il collegio uninominale della Valle d'Aosta e le ripartizioni Estero, la palma spetta invece al PD, che ha sopravanzato il M5S di quattro decimi di punto, pari a circa 135 mila voti, e di 4 punti percentuali il PDL, nei confronti del quale si avvantaggia per poco meno di un milione e mezzo di voti). Come si può osservare nella tabella, il movimento di Grillo è stato l'opzione più votata in sei circoscrizioni: le siciliane, Sardegna, Marche, Liguria, Abruzzo. È inoltre il partito più votato in 50 province su 109 (contro 40 per il PD e 17 per il PDL). I suoi risultati peggiori sono stati quelli di Lombardia 2 e Trentino-Alto Adige, circoscrizioni in cui è rimasto sotto al $20 \%$ dei voti validi.

L'altra new entry, la coalizione guidata da Monti, ha invece ottenuto un risultato al di sotto delle aspettative. Ha superato di poco la soglia del 10\% e soltanto Scelta civica, che ha evidentemente cannibalizzato i suoi alleati, ha ricevuto più del $4 \%$ dei voti. Questo risultato ha escluso dalla Camera FLI, il partito di Fini, poiché ad avvalersi della clausola del miglior perdente è stato l'UDC, che ha ottenuto 8 seggi (si torni alla $\mathrm{TAB} .7$ e si veda l'Appendice). La coalizione Monti ha ottenuto i suoi migliori risultati nelle circoscrizioni lombarde e venete, in Friuli Venezia Giulia e Piemonte 2, arrivando a sfiorare il 14\% nel Trentino-Alto Adige (grazie al $20 \%$ della provincia di Trento ottenuto per propulsione di Lorenzo Dellai). Lo squilibrio territoriale è confermato dai risultati inferiori o di poco superiori al 10\% relativi alle regioni rosse e alle circoscrizioni del Sud (uniche eccezioni: Campania 2 e Basilicata, dove la coalizione Monti ha superato la media nazionale).

Nelle circoscrizioni dell'Italia del premio, la configurazione multipolare della com- 


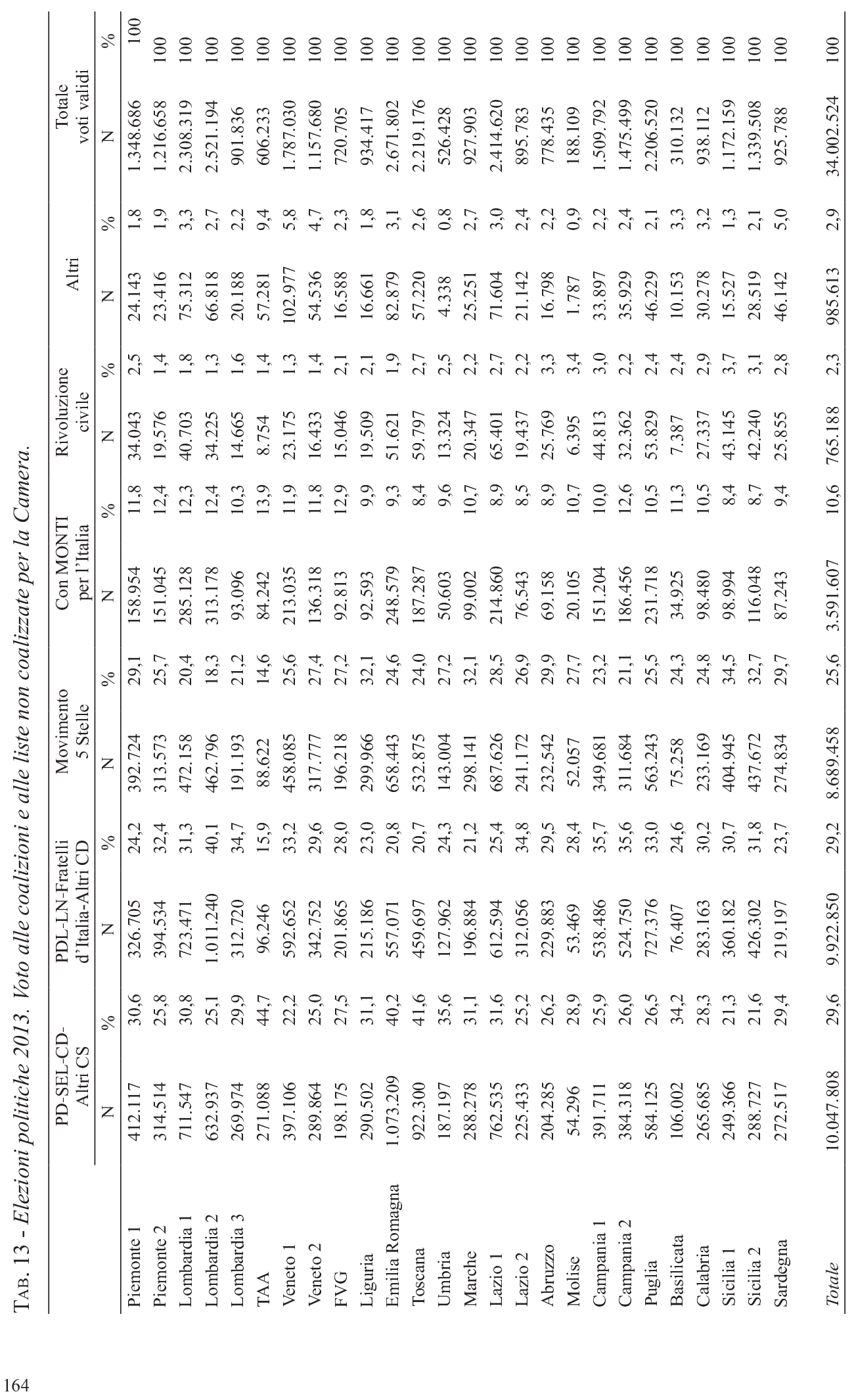


petizione ha prodotto una distribuzione dei voti molto diversa da quella del 2008 e del 2006. Soltanto in quattro circoscrizioni la coalizione o lista non coalizzata che vince ha oltrepassato la soglia del 40\% dei voti (in Toscana, Emilia Romagna e Trentino Alto Adige il centro-sinistra, in Lombardia 1 il centro-destra). In cinque circoscrizioni (Veneto 2, Friuli-Venezia Giulia, Abruzzo, Molise e Sardegna) nessuna opzione ha superato il 30\%.

La TAB. 14 riporta il risultato della circoscrizione Valle d'Aosta. Come si è ricordato, si tratta di un collegio uninominale che non concorre al processo di assegnazione dei seggi su scala nazionale e alla determinazione delle grandezze per l'attribuzione del(l'eventuale) premio di maggioranza. Il candidato del partito autonomista Vallée d'Aoste, Marguerettaz, ha conquistato il seggio con un vantaggio di tre decimi di punto (meno di 200 voti) sul candidato dell'UVP, Vierin (centro-sinistra). Si è trattato di un margine ancora più piccolo di quello, già modesto, che nel 2008 aveva determinato la sconfitta della candidatura di Vallée d'Aoste a vantaggio della candidatura dell'ALD. La lista autonomista ha vinto anche al Senato, con un margine più consistente (si veda la TAB. 15). La Valle d'Aosta è l'unica circoscrizione in cui il M5S ottiene più voti al Senato che alla Camera.

TAв. 14 - Elezioni politiche 2008, Camera. Risultato della circoscrizione uninominale della Valle d'Aosta.

\begin{tabular}{|c|c|c|c|}
\hline \multirow[t]{2}{*}{ Candidati } & \multirow[t]{2}{*}{ Partiti } & \multicolumn{2}{|c|}{ Voti } \\
\hline & & $\mathrm{N}$. & $\%$ \\
\hline Rudi Franco Marguerettaz & Vallée d'Aoste & 18.376 & 25,4 \\
\hline Laurent Vierin & Union Valdôtaine Progressiste & 18.191 & 25,1 \\
\hline Jean Pierre Guichardaz & Autonomie Liberté Démocratie & 14.340 & 19,8 \\
\hline Roberto Ugo Massimo Cognetta & M5S & 13.403 & 18,5 \\
\hline Giorgia Meloni & Fratelli d'Italia & 3.051 & 4,2 \\
\hline Nicoletta Spelgatti & Lega Nord & 2.384 & 3,3 \\
\hline Luca Bringhen & UDC & 1.355 & 1,9 \\
\hline Fabrizio Buillet & Fare per fermare il declino & 748 & 1,0 \\
\hline Andrea Ladu & CasaPound Italia & 443 & 0,6 \\
\hline Eros Campion & NAtion Val d'Outa & 145 & 0,2 \\
\hline Totale & & 72.436 & \\
\hline
\end{tabular}

In Trentino-Alto Adige, dove i senatori della regione vengono eletti con il sistema misto vigente dal 1993 (legge Mattarella), il centro-sinistra ha conquistato sei seggi su sette (si veda la TАВ. 16). Grazie all'asse PD-SVP in Alto Adige e all'accordo tra il PD e l'UPT di Dellai in provincia di Trento, lo schieramento ha conquistato tutti i collegi uninominali, compresi due collegi tradizionalmente marginali come quelli di Bolzano e Rovereto. Al centro-destra è andato il seggio dell'arena proporzionale. 
TAв. 15 - Elezioni politiche 2013, Senato. Risultato della circoscrizione uninominale della Valle d'Aosta.

\begin{tabular}{llrr}
\hline Candidati & & \multicolumn{2}{c}{ Partiti } \\
\cline { 3 - 4 } & & \multicolumn{2}{c}{ N. } \\
\hline Albert Laniece & Vallée d'Aoste & 24.609 & 37,0 \\
Patrizia Morelli & Autonomie Liberté Démocratie & 20.430 & 30,8 \\
Stefano Angelo Luigi Ferrero & M5S & 13.760 & 20,7 \\
Sandra Maria Cane & Lega Nord & 2.608 & 3,9 \\
Monza Paolo Dalbard & La Destra & 2.014 & 3,0 \\
Luigi Bracci & UDC & 1.594 & 2,4 \\
Enrico Martial & Fare per fermare il declino & 814 & 1,2 \\
Vilma Margaria & CasaPound Italia & 424 & 0,6 \\
Giovan Battista Mascia & Nation Val D'Outa & 186 & 0,3 \\
& & & \\
Totale & & 66.439 & 100 \\
\hline
\end{tabular}

ТАв. 16 - Elezioni politiche 2013, Senato. Risultato della circoscrizione Trentino Alto-Adige.

\begin{tabular}{lrrr}
\hline & \multicolumn{2}{c}{ Voti } & Seggi \\
\cline { 2 - 4 } & Partiti & $\%$ & N. \\
\hline & & & 3 \\
SVP - PATT - PD - UPT & 127.656 & 23,4 & 1 \\
PDL-Lega Nord & 85.298 & 15,7 & 1 \\
M5S & 82.499 & 15,2 & 1 \\
SVP & 54.474 & 10,0 & 1 \\
PD - SVP & 47.623 & 8,8 & \\
SVP & 42.667 & 7,8 & \\
Die Freiheitlichen & 42.094 & 7,7 & \\
Rivoluzione civile & 11.262 & 2,1 & \\
Fare per fermare il declino & 8.796 & 1,6 & \\
Verdi-Grüne-Verc & 6.686 & 1,2 & \\
Verdi-Grüne-Verc & 6.122 & 1,1 & \\
L'Altro Adige nel cuore & 4.672 & 0,9 & \\
PD & 4.478 & 0,8 & \\
PD & 4.319 & 0,8 & \\
Scelta civica con Monti per l'Italia & 4.016 & 0,7 & \\
Scelta civica con Monti per l'Italia & 3.630 & 0,7 & \\
MIR - Moderati in rivoluzione & 3.414 & 0,6 & \\
Fratelli d'Italia & 2.365 & 0,4 & \\
La Destra & & 0,2 & \\
CasaPound Italia & 1.160 & 0,2 & \\
Partito per tutti-Partei für alle & 426 & 0,1 & \\
Totale & & & \\
\hline
\end{tabular}


La ТАВ. 17 riporta il riepilogo dei risultati nelle circoscrizioni regionali del Senato con riferimento alle coalizioni e alle liste non coalizzate. La struttura della competizione presenta alcune variazioni minori rispetto a quella della Camera, in particolare una dispersione del voto lievemente più contenuta (al Senato la somma delle prime due coalizioni è pari al $62,3 \%$ contro il $58,8 \%$ della Camera e sulle prime tre opzioni all' $86,1 \%$ contro $1^{\prime} 84,4 \%$ ). Ciò nonostante, la coalizione o lista non coalizzata vincente ha oltrepassato la soglia del $40 \%$ dei voti in due sole circoscrizioni (Toscana e Emilia Romagna). Sono invece tre le regioni in cui il vincente non raggiunge il 30\% (Piemonte, Friuli-Venezia Giulia e Abruzzo). A differenza della Camera, il M5S non ha conquistato il maggior numero di voti in nessuna circoscrizione e dunque non ottiene alcun premio di maggioranza. Ha superato il 30\% soltanto in Liguria e nelle Marche, mentre in Lombardia è rimasto sotto al $20 \%$ dei voti. Anche l'opzione Monti, in corsa al Senato come lista unica, ha registrato nel voto per l'assemblea di Palazzo Madama risultati peggiori di quelli ricevuti per la Camera. Il suo miglior risultato è conseguito in Friuli Venezia Giulia (12,3\%). In Piemonte e Veneto ha superato 1'11\%. Come si è visto nella TAB. 11, la lista Con Monti per l'Italia è rimasta sotto la soglia dell' $8 \%$ in 5 circoscrizioni e questo ne ha indebolito il contingente in seggi.

La distribuzione dei seggi assegnate in ciascuna regione alle coalizione e alle liste (coalizzate e non coalizzate) è riportata nella $\mathrm{TAB}$. 18. È importante osservare in che modo le due coalizioni principali - la coalizione Bersani e la coalizione Berlusconi - si sono divisi i 17 premi di maggioranza, fattore determinante del risultato delle elezioni. Il centro-sinistra ne ha ottenuti di più ( 10 contro i 7 andati al centro-destra), ma il centrodestra ha conquistato, con la sola eccezione del Lazio, i premi più "pesanti", ossia quelli assegnati nelle circoscrizioni più grandi per seggi in palio: Lombardia, Campania, Sicilia e Veneto.

Il confronto 2013-2008: milioni di voti in movimento, volatilità senza precedenti, prospettive incerte

Da quanto si è osservato nei paragrafi precedenti, il risultato delle politiche 2013 ha rivoluzionato il paesaggio elettorale e parlamentare. Si ricorderà che anche le elezioni del 2008 erano state di grande cambiamento elettorale. Ne era scaturito un paesaggio politico a bassa frammentazione e incentrato sui due nuovi partiti che si erano formati poco prima del voto, il PD e il PDL. Ancora una volta, dunque, settori assai consistenti di elettorato hanno modificato la propria scelta di voto rispetto all'elezione precedente, a conferma di una diagnosi di fluidità e destrutturazione del sistema dei partiti ormai ventennale.

Un indicatore grezzo dell'entità di questo fenomeno si può ricavare confrontando i voti delle forze in campo con i risultati del 2008. I saldi riportati nella TAB. 19 mostrano l'entità del sommovimento. Occorre considerare, anzitutto che, rispetto al 2008, i voti validi sono stati due milioni e mezzo in meno. Le elezioni politiche del 2013, in secondo luogo, hanno assistito al fragoroso ingresso sulla scena di movimenti e partiti al 


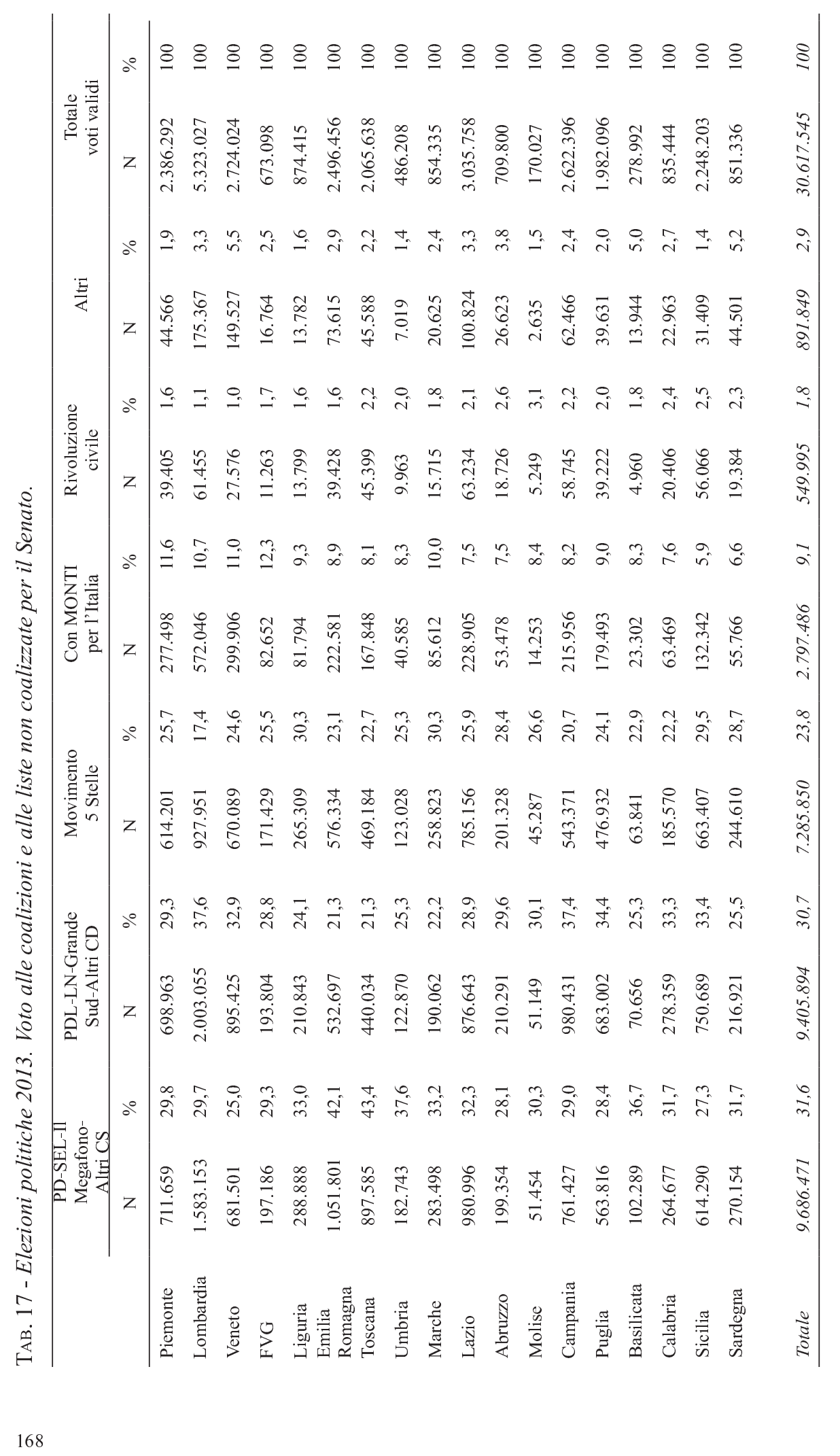




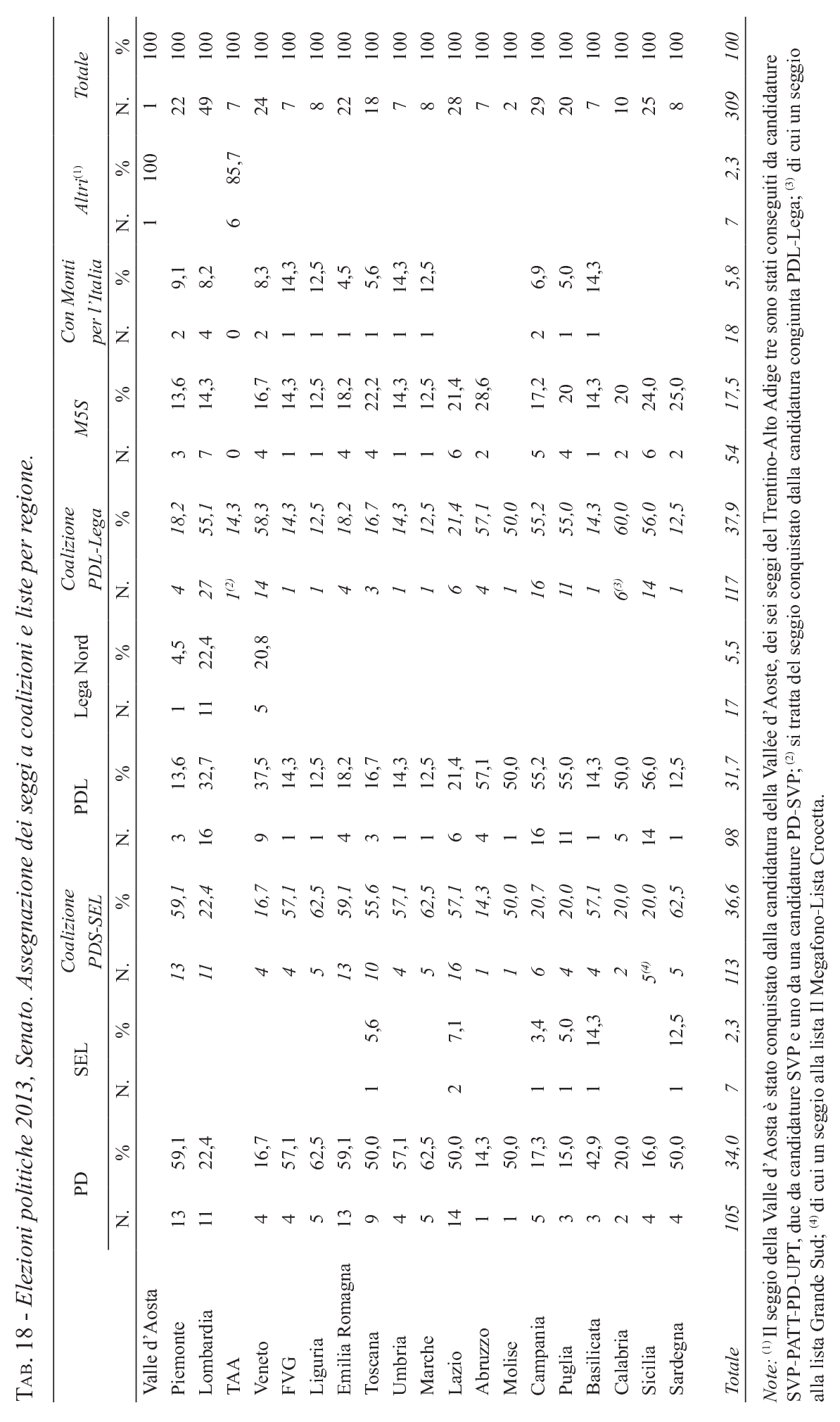


loro esordio. Uno di essi, il M5S, è stato votato da oltre 8 milioni e mezzo di elettori che nelle elezioni precedenti si erano astenuti o avevano votato per un partito diverso. Pur se di minore impatto, si è avuto inoltre l'esordio di Scelta civica, lista creata dal premier uscente Monti, che ha ricevuto quasi 3 milioni di voti.

A questi risultati hanno corrisposto i saldi negativi dei partiti che nel 2008 avevano avuto un seguito elettorale assai consistente. Il PDL ha perduto oltre 6 milioni di voti, risultato senza precedenti nella storia repubblicana (lo scarto tra PPI 1994 e DC 1992 è superiore, ma politicamente spurio). In termini percentuali la Lega Nord ha fatto ancor peggio, perdendo oltre la metà dei voti conseguiti cinque anni prima. Il PD ha subito una flessione minore, ma ha perso pur sempre poco meno di 3 milioni e mezzo di voti, pari a quasi il $30 \%$ del proprio elettorato precedente. Si segnala anche il prosciugamento dell'UDC di Casini che vede evaporare il voto di due elettori su tre che nel 2008 avevano scelto lo Scudo crociato. Parte di questi elettori sembra essersi rivolta a Scelta civica. C'è infine l'estrema sinistra, che nel 2008 era uscita assai malconcia dal voto e che ha visto ridursi ancora di un terzo il proprio seguito.

TAB. 19 - il cambiamento del sostegno elettorale ai partiti tra politiche 2013 e politiche 2008 (Camera).

\begin{tabular}{|c|c|c|c|c|}
\hline & \multicolumn{3}{|c|}{ Voti } & \multirow{2}{*}{$\begin{array}{l}\text { Voti persi } \\
(2008=100)\end{array}$} \\
\hline & 2013 & 2008 & $\begin{array}{c}\Delta \\
(2013-2008)\end{array}$ & \\
\hline Voti validi & 34.002 .524 & 36.457 .254 & -2.454 .730 & $-6,7$ \\
\hline PDL & 7.332 .972 & 13.629 .464 & -6.296 .492 & $-46,2$ \\
\hline Lega Nord & 1.390 .014 & 3.024 .543 & -1.634 .529 & $-54,0$ \\
\hline PD & 8.644 .523 & 12.095 .306 & -3.450 .783 & $-28,6$ \\
\hline UDC & 608.210 & 2.050 .229 & -1.442 .019 & $-70,3$ \\
\hline Estrema sinistra $^{(*)}$ & 1.854 .597 & 2.718 .322 & -863.725 & $-31,8$ \\
\hline M5S & 8.689 .458 & & +8.689 .458 & \\
\hline Scelta civica & 2.824 .065 & & +2.824 .065 & \\
\hline
\end{tabular}

(*) Nel 2013: SEL e Rivoluzione civile Nel 2008 IDV e Sinistra arcobaleno.

La TAB. 20 approfondisce su scala circoscrizionale il confronto 2013-2008, con riferimento ai quattro partiti che hanno e avevano ottenuto rappresentanza parlamentare. Se ne ricavano alcune indicazioni sulla geografia della loro pesante flessione. Il PDL ha perso in cinque circoscrizioni più della metà dei voti che aveva ricevuto nel 2008. Si tratta, in ordine decrescente, di Sardegna, Basilicata, Marche, Liguria e Lazio 1. Ha invece contenuto le sue perdite nelle roccaforti venete e lombarde (ma non in Lombardia 1), dove ha comunque perso più di un terzo dei propri voti precedenti. Al Sud il PDL è in grande calo soprattutto nelle circoscrizioni siciliane e in Campania 2, meno in Puglia e Campania 1. Anche l'alleato leghista ha registrato un tracollo inferiore alla media - pur se compreso tra il $40 \%$ e il $50 \%$ dei propri voti precedenti - nelle circoscrizioni lombarde. Ha registrato invece le maggiori 


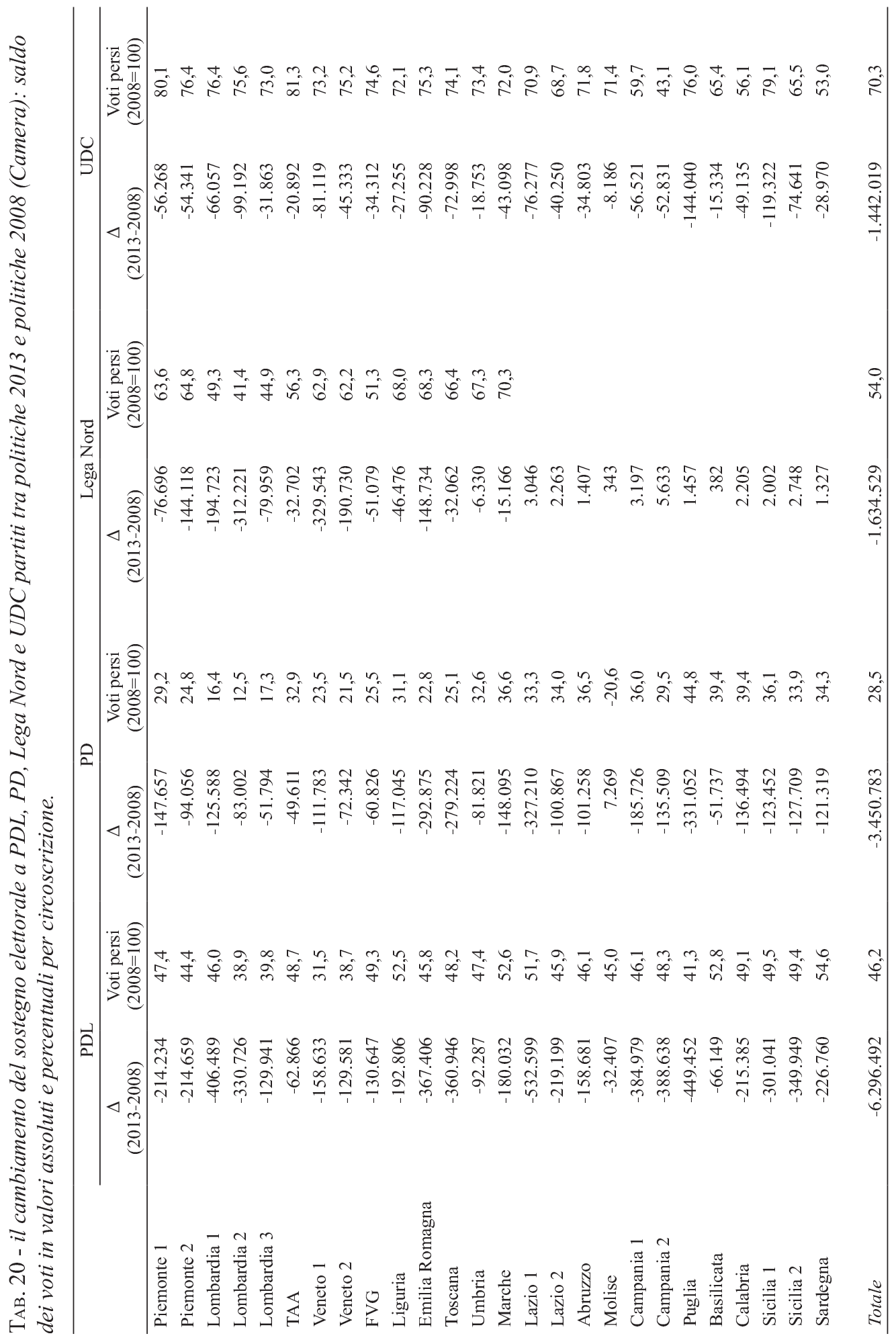


perdite nelle regioni di più recente insediamento, ossia le regioni rosse e la Liguria. Nelle due regioni di cui guida il governo, Piemonte e Veneto, il bilancio è stato pesante, con una flessione di poco inferiore ai due terzi del proprio elettorato precedente.

Il PD, come si è visto, ha perso meno dei partiti di centro-destra. L'andamento di tali flessioni presenta un profilo geografico più definito. Con l'eccezione di tre circoscrizioni - nell'ordine Marche, Liguria, Umbria e Piemonte 1 - le perdite superiori alla media sono concentrate a sud di Roma (fa eccezione il Molise, dove il PD ottiene addirittura un saldo positivo legato peraltro al magro risultato di cinque anni prima, a sua volta dovuto al pieno di voti realizzato dall'IDV del molisano Di Pietro, allora alleato del PD). Il partito di Bersani ha invece contenuto le sue perdite sia nelle regioni di maggior radicamento (Emilia Romagna e Toscana, dove perde "soltanto" un quarto dei propri voti precedenti), sia nelle circoscrizioni di maggior debolezza, quelle lombarde e venete.

La geografia del virtuale azzeramento dell'UDC è anch'essa piuttosto connotata, chiamando in causa soprattutto le zone di forza del Nord (le due circoscrizioni piemontesi e il Veneto 2) più Sicilia 1 e Puglia. In queste zone l'UDC ha perso oltre i tre quarti del proprio elettorato precedente. Ha resistito meglio in alcune delle sue roccaforti al Sud, in particolare le circoscrizioni campane, la Calabria, Sicilia 2 e Lazio 2.

\section{Il voto degli italiani all'estero}

Per la terza volta gli italiani residenti all'estero hanno eletto i 18 parlamentari (12 deputati e sei senatori) che li rappresentano nel parlamento di Roma. Al voto erano iscritti circa tre milioni e mezzo di elettori.

TAв. 21 - Elezioni Politiche 2013, Camera. Elettori, votanti e voti validi, nelle quattro ripartizioni Estero.

\begin{tabular}{lccccccc}
\hline & Elettori & Votanti & $\begin{array}{c}\% \\
\text { Votanti }\end{array}$ & $\begin{array}{c}\text { Voti } \\
\text { validi }\end{array}$ & $\begin{array}{c}\text { Schede } \\
\text { nulle }\end{array}$ & $\begin{array}{c}\text { Schede } \\
\text { bianche }\end{array}$ & $\begin{array}{c}\text { Schede } \\
\text { contestate }\end{array}$ \\
\hline Europa & 1.886 .645 & 577.631 & 30,6 & 521.824 & 47.592 & 7.640 & 575 \\
America del Sud & 1.093 .766 & 374.157 & 34,2 & 330.682 & 35.310 & 7.089 & 1.076 \\
America del Nord & 328.788 & 96.434 & 29,3 & 82.374 & 12.314 & 981 & 765 \\
Asia, Africa, & 185.488 & 55.767 & 30,1 & 47.447 & 7.544 & 746 & 30 \\
$\begin{array}{l}\text { Oceania e Antartide } \\
\text { Totale }\end{array}$ & 3.494 .687 & 1.103 .989 & 31,6 & 982.327 & 102.760 & 16.456 & 2.446 \\
\hline
\end{tabular}

Come si legge nelle ТАВв. 21 e 22 , ha votato poco più del $30 \%$ degli aventi diritto, con una caduta di partecipazione rispetto al 2008 di circa 10 punti. Se a ciò si aggiungono il $10 \%$ di voti nulli, il rapporto tra voti validi ed elettori si riduce al $28 \%$. Si tratta di una percentuale assai modesta, che non sembra giustificare la molta retorica e l'astrusa complessità che circonda l'anomalo meccanismo di voto introdotto nel 2001 con riforma costituzionale. I risultati - si vedano le TABB. 23 e 24 - hanno premiato il PD, che conquista la metà dei seggi in palio ( 9 su 18,5 alla Camera e 4 al Senato) probabil- 
mente grazie alla maggiore attenzione applicata su tali circoscrizioni (il centro-sinistra ha riconfermato tre uscenti e un quarto eletto era stato in carica nella XV legislatura). Il centro-destra ha invece proseguito la sua serie di risultati negativi e ha ottenuto un solo seggio, nella ripartizione Europa per la Camera. Nelle circoscrizioni estere non ha sfondato il M5S che, rimasto sotto il 10\%, ha eletto un solo parlamentare, anch'esso in Europa alla Camera. Ha conseguito invece un buon risultato Scelta civica, che ha conquistato due seggi alla Camera e uno al Senato. In Sudamerica ha trovato conferma l'attivismo dell'associazionismo di origine italiana. Il MAIE è riuscito a eleggere tre parlamentari, due alla Camera e uno al Senato, ai quali si aggiunge l'eletto dell'USEI.

TAв. 22 - Elezioni Politiche 2013, Senato. Elettori, votanti e voti validi, non validi nelle quattro ripartizioni Estero.

\begin{tabular}{lccccccc}
\hline & Elettori & Votanti & $\begin{array}{c}\% \\
\text { Votanti }\end{array}$ & $\begin{array}{c}\text { Voti } \\
\text { validi }\end{array}$ & $\begin{array}{c}\text { Schede } \\
\text { nulle }\end{array}$ & $\begin{array}{c}\text { Schede } \\
\text { bianche }\end{array}$ & $\begin{array}{c}\text { Schede } \\
\text { contestate }\end{array}$ \\
\hline Europa & 1.688 .574 & 533.898 & 31,6 & 479.339 & 46.889 & 7.419 & 251 \\
America del Sud & 984.045 & 334.075 & 33,9 & 293.998 & 33.333 & 6.554 & 190 \\
America del Nord & 306.750 & 90.230 & 29,4 & 77.717 & 11.554 & 936 & 23 \\
Asia, Africa, & 170.132 & 51.718 & 30,4 & 44.105 & 6.889 & 705 & 19 \\
Oceania e Antartide & & & & & & & \\
Totale & 3.149 .501 & 1.009 .921 & 32,1 & 895.159 & 98.665 & 15.614 & 483 \\
\hline
\end{tabular}

ТАв. 23 - Elezioni Politiche 2013, Camera. Voti e seggi alle liste nelle quattro ripartizioni Estero.

\begin{tabular}{|c|c|c|c|c|c|c|c|c|c|c|}
\hline & \multicolumn{2}{|c|}{ Europa } & \multicolumn{2}{|c|}{ America del Sud } & \multicolumn{2}{|c|}{$\begin{array}{l}\text { America del } \\
\text { Nord }\end{array}$} & \multicolumn{2}{|c|}{$\begin{array}{c}\text { Asia, Africa, } \\
\text { Oceania, Antartide }\end{array}$} & \multicolumn{2}{|c|}{ Totale } \\
\hline & Voti & Seggi & Voti & Seggi & Voti & Seggi & Voti & Seggi & Voti & Seggi \\
\hline PD & 154.871 & 2 & 90.716 & 1 & 26.710 & 1 & 15.795 & 1 & 288.092 & 5 \\
\hline $\begin{array}{l}\text { Con Monti per } \\
\text { l'Italia }\end{array}$ & 142.980 & 1 & & & 22.895 & 1 & 14.799 & & 180.674 & 2 \\
\hline PDL & 95.262 & 1 & 21.809 & & 18.772 & & 9.981 & & 145.824 & 1 \\
\hline $\begin{array}{l}\text { MAIE - Mov. } \\
\text { Associativo Italiani } \\
\text { all'estero }\end{array}$ & 10.642 & & 129.831 & 2 & & & & & 140.473 & 2 \\
\hline M5S & 69.284 & 1 & 10.511 & & 8.374 & & 6.872 & & 95.041 & 1 \\
\hline $\begin{array}{l}\text { USEI-Unione } \\
\text { Sudamericana } \\
\text { Emigrati Italiani }\end{array}$ & & & 44.024 & 1 & & & & & 44.024 & 1 \\
\hline $\begin{array}{l}\text { Italiani per la } \\
\text { libertà }\end{array}$ & & & 22.321 & & & & & & 22.321 & \\
\hline SEL & 17.375 & & & & & & & & 17.375 & \\
\hline Rivoluzione civile & 15.910 & & & & & & & & 15.910 & \\
\hline $\begin{array}{l}\text { Unione italiani } \\
\text { Sudamerica }\end{array}$ & & & 11.470 & & & & & & 11.470 & \\
\hline $\begin{array}{l}\text { Fare per fermare il } \\
\text { declino }\end{array}$ & 8.427 & & & & 1.733 & & & & 10.160 & \\
\hline Partito comunista & 7.073 & & & & & & & & 7.073 & \\
\hline $\begin{array}{l}\text { Insieme per gli } \\
\text { Italiani }\end{array}$ & & & & & 3.890 & & & & 3.890 & \\
\hline Totale generale & 521.824 & 5 & 330.682 & 4 & 82.374 & 2 & 47.447 & 1 & 982.327 & 12 \\
\hline
\end{tabular}


TAB. 24 - Elezioni Politiche 2013, Senato. Voti e seggi alle liste nelle quattro ripartizioni Estero.

\begin{tabular}{|c|c|c|c|c|c|c|c|c|c|c|}
\hline & \multicolumn{2}{|c|}{ Europa } & \multicolumn{2}{|c|}{ America del Sud } & \multicolumn{2}{|c|}{$\begin{array}{l}\text { America del } \\
\text { Nord }\end{array}$} & \multicolumn{2}{|c|}{$\begin{array}{c}\text { Asia, Africa, } \\
\text { Oceania, Antartide }\end{array}$} & \multicolumn{2}{|c|}{ Totale } \\
\hline & Voti & Seggi & Voti & Seggi & Voti & Seggi & Voti & Seggi & Voti & Seggi \\
\hline PD & 154.074 & 1 & 79.694 & 1 & 26.332 & 1 & 14.632 & 1 & 274.732 & 4 \\
\hline $\begin{array}{l}\text { Con Monti per } \\
\text { l'Italia }\end{array}$ & 142.207 & 1 & & & 22.437 & & 12.758 & & 177.402 & 1 \\
\hline PDL & 88.322 & & 20.130 & & 17.547 & & 10.053 & & 136.052 & 0 \\
\hline $\begin{array}{l}\text { MAIE - Mov. } \\
\text { Associativo } \\
\text { Italiani all'estero }\end{array}$ & & & 120.290 & 1 & & & & & 120.290 & 1 \\
\hline M5S & 65.132 & & 9.590 & & 8.178 & & 6.662 & & 89.562 & 0 \\
\hline $\begin{array}{l}\text { USEI-Unione } \\
\text { Sudamericana } \\
\text { Emigrati Italiani }\end{array}$ & & & 38.223 & & & & & & 38.223 & 0 \\
\hline $\begin{array}{l}\text { Italiani per la } \\
\text { libertà }\end{array}$ & & & 15.260 & & & & & & 15.260 & 0 \\
\hline $\begin{array}{l}\text { Rivoluzione } \\
\text { civile }\end{array}$ & 14.134 & & & & & & & & 14.134 & 0 \\
\hline $\begin{array}{l}\text { Unione italiani } \\
\text { Sudamerica }\end{array}$ & & & 10.811 & & & & & & 10.811 & 0 \\
\hline $\begin{array}{l}\text { Fare per fermare } \\
\text { il declino }\end{array}$ & 7.892 & & & & & & & & 7.892 & 0 \\
\hline Partito comunista & 7.578 & & & & & & & & 7.578 & 0 \\
\hline $\begin{array}{l}\text { Insieme per gli } \\
\text { Italiani }\end{array}$ & & & & & 3.223 & & & & 3.223 & 0 \\
\hline Totale generale & 479.339 & 2 & 293.998 & 2 & 77.717 & 1 & 44.105 & 1 & 895.159 & 6 \\
\hline
\end{tabular}




\section{APPENDICE}

Riepilogo generale dei risultati delle elezioni per la Camera dei deputati e per il Senato della Repubblica del 24-25 febbraio 2013 


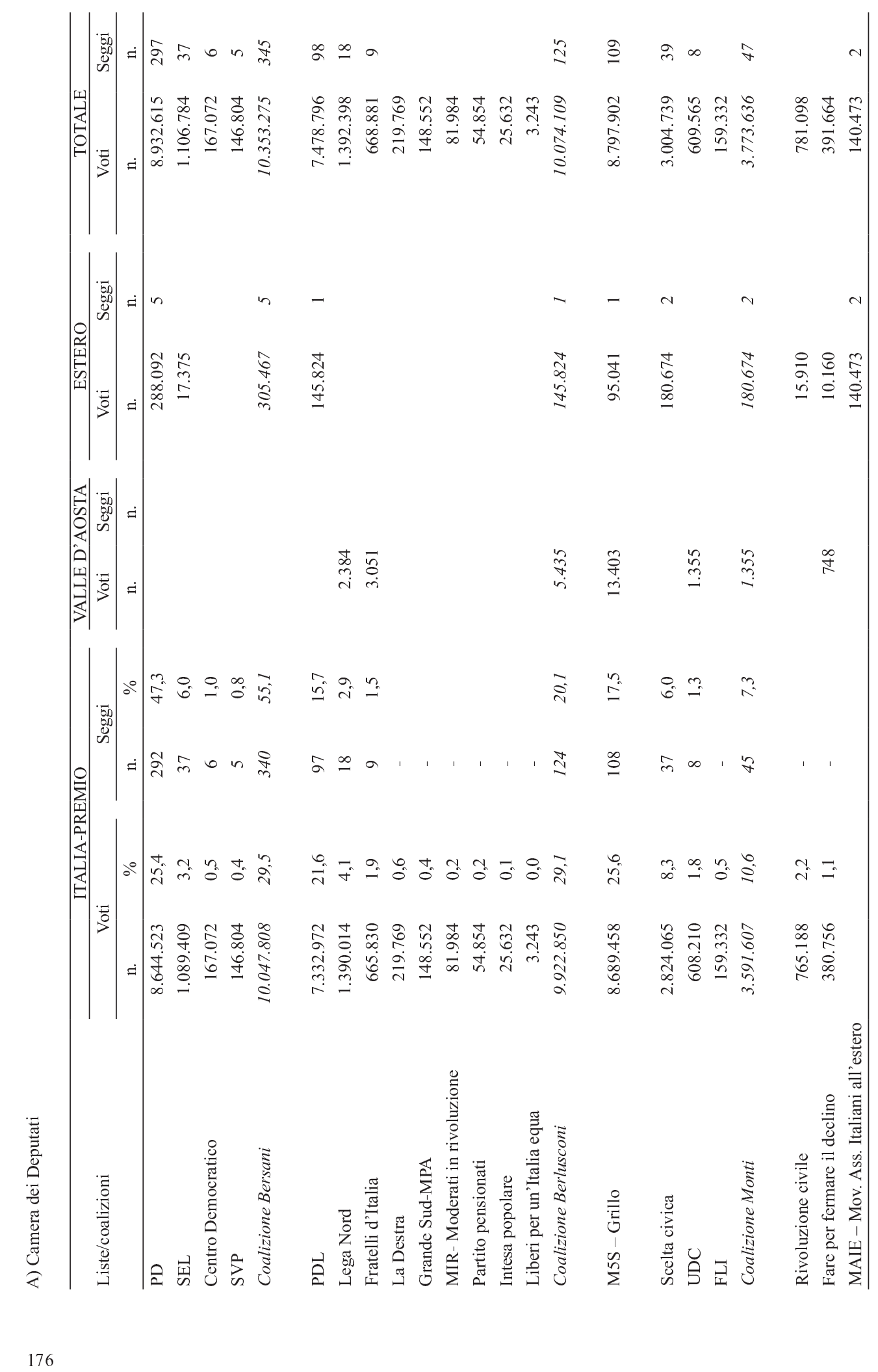




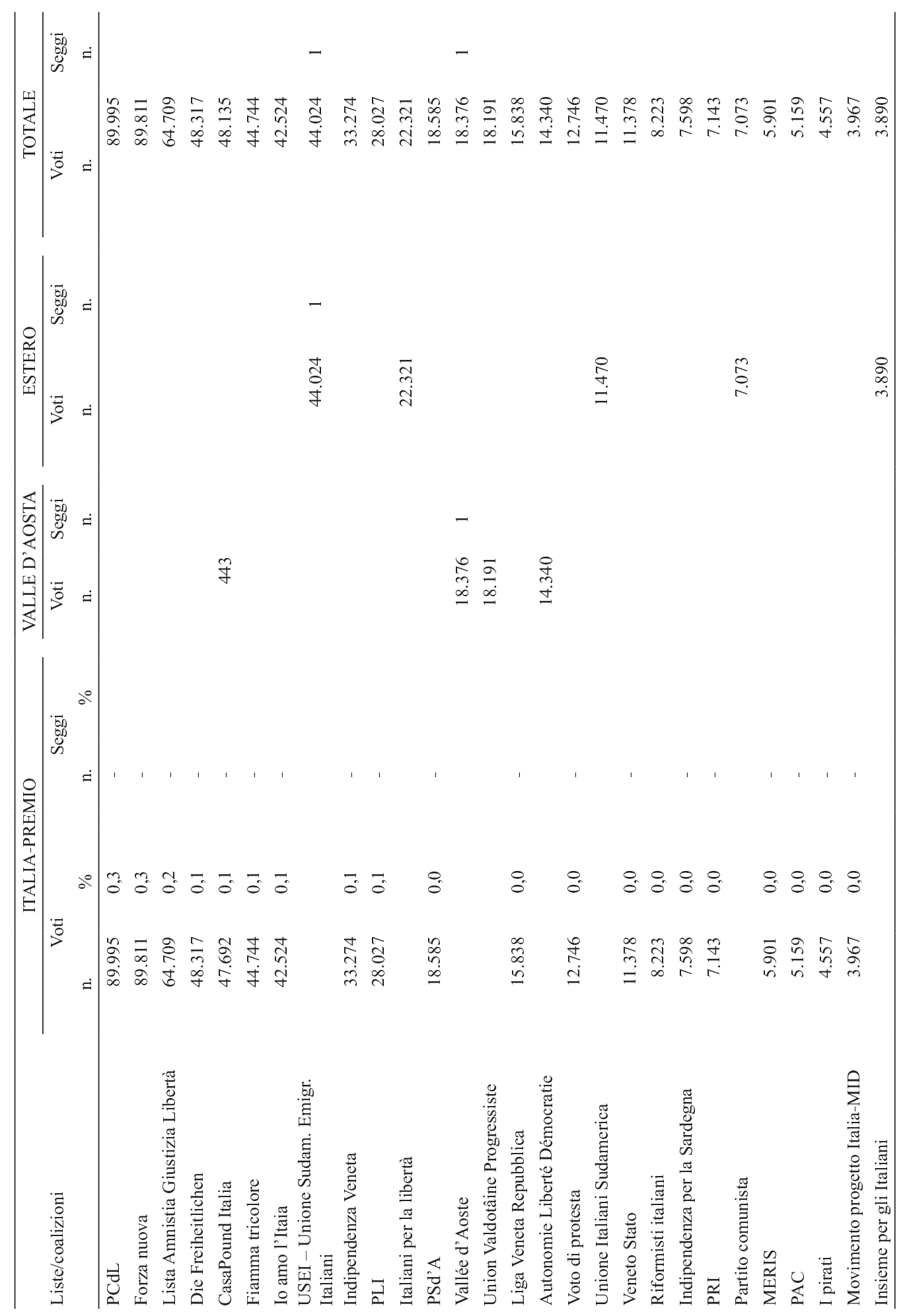




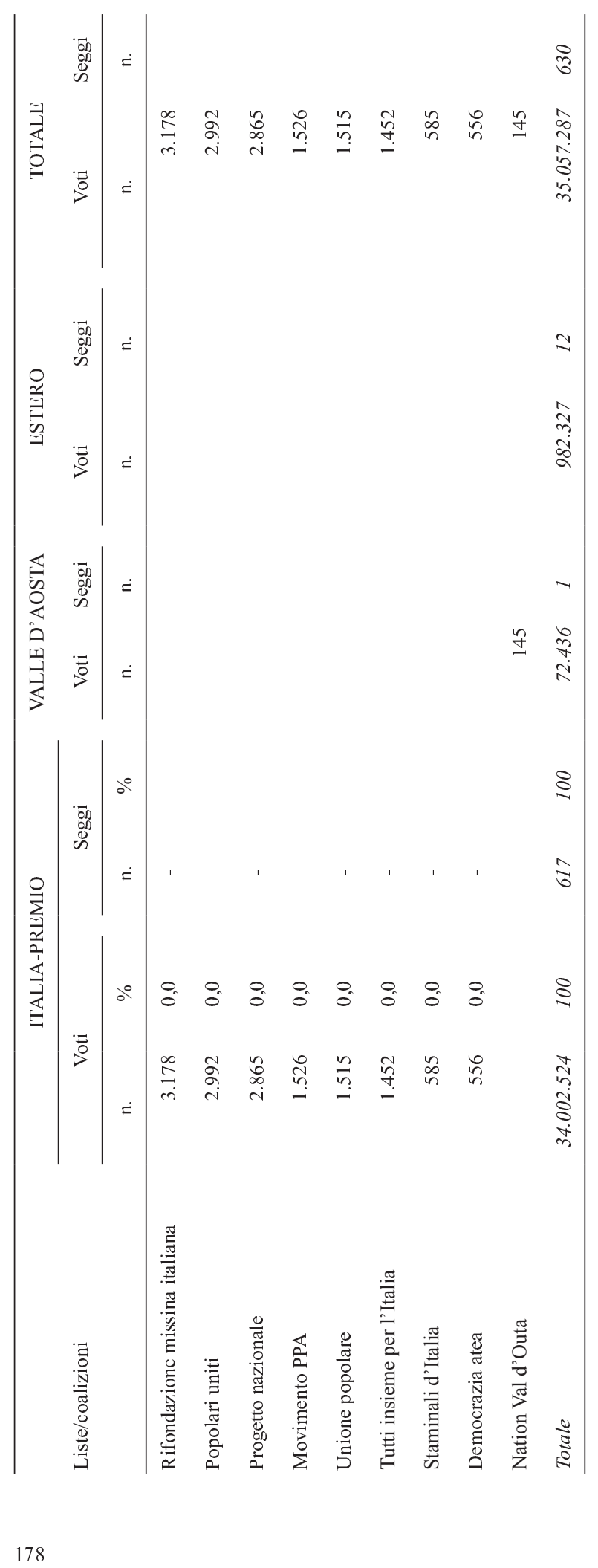




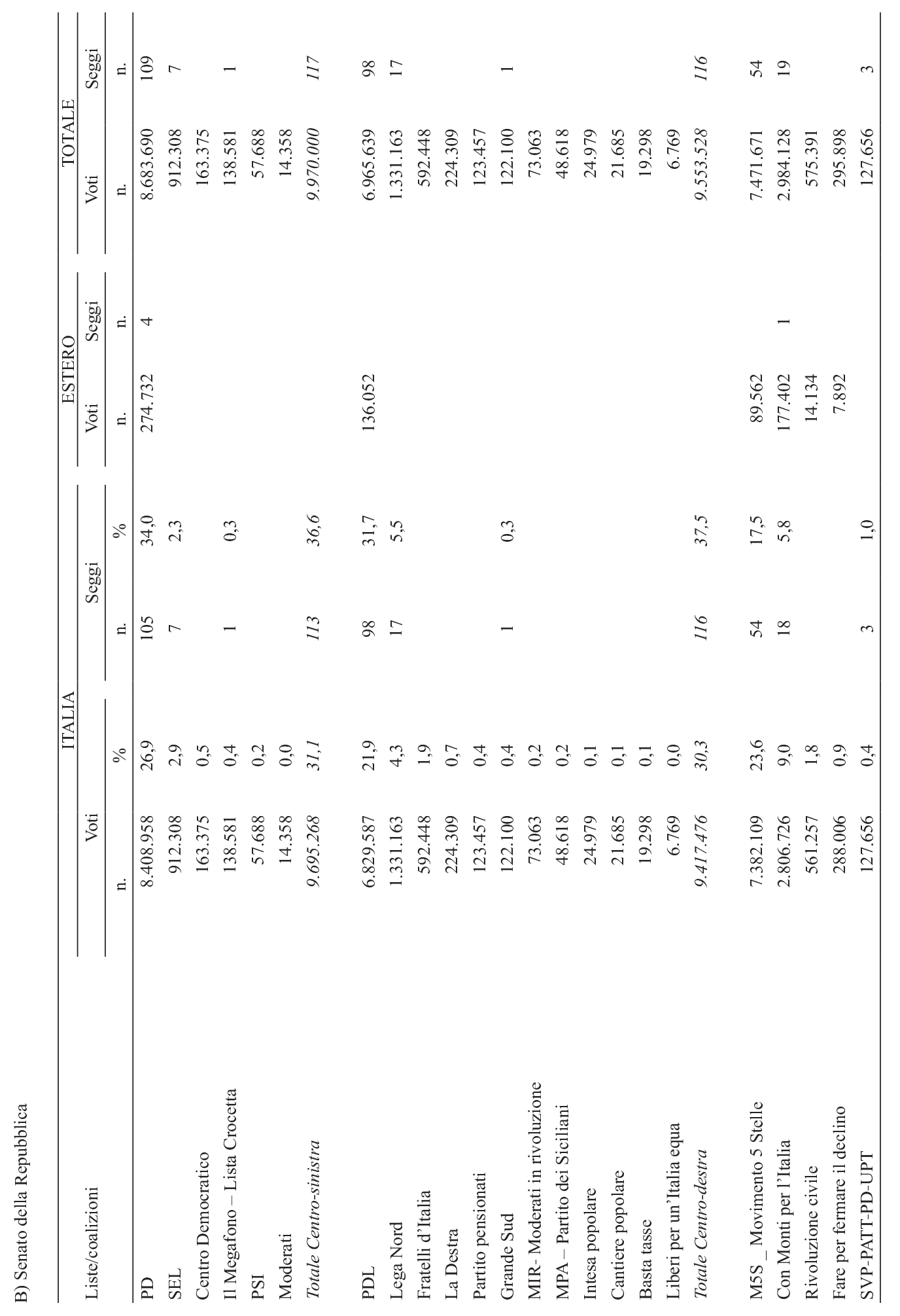




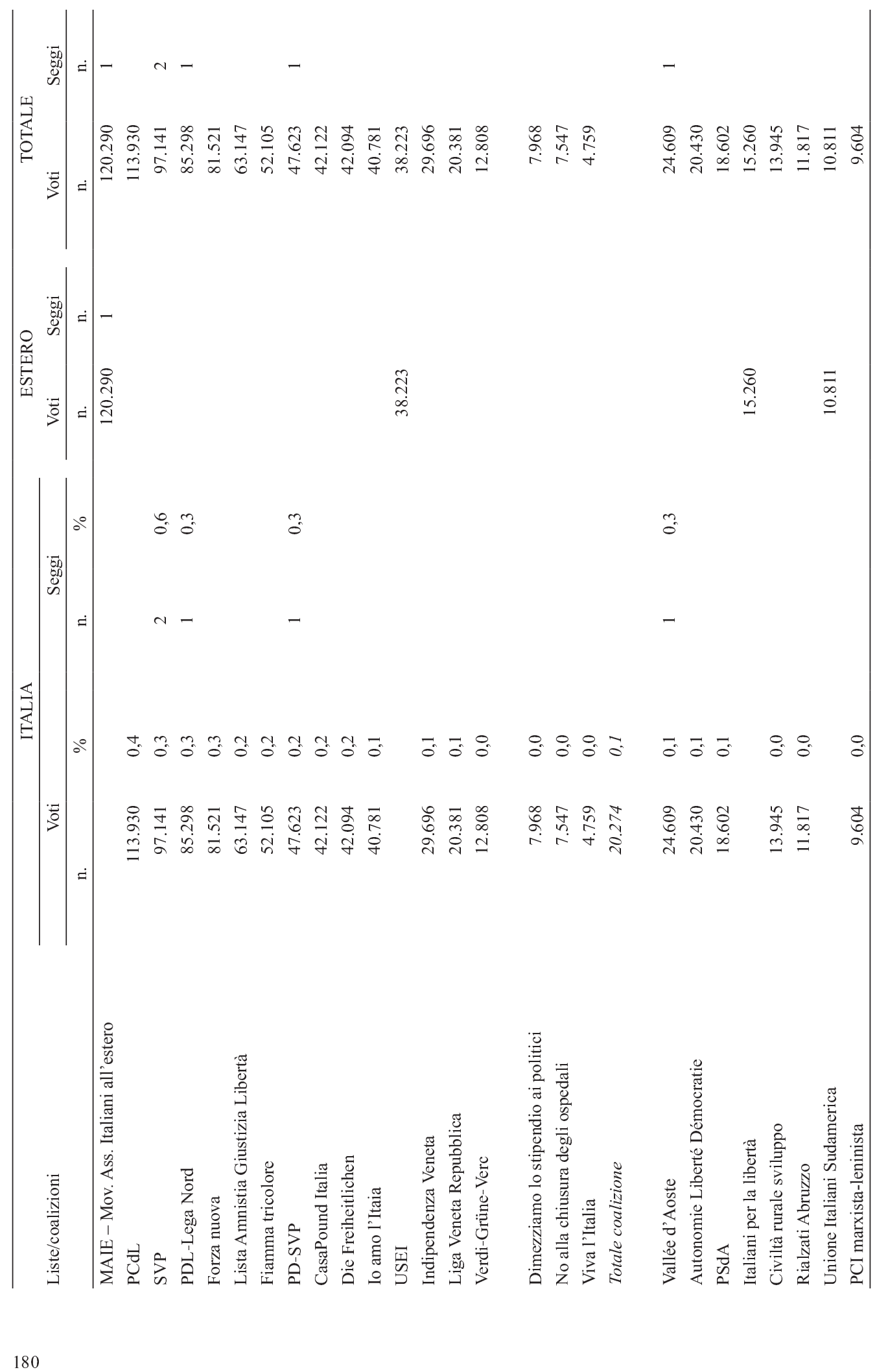




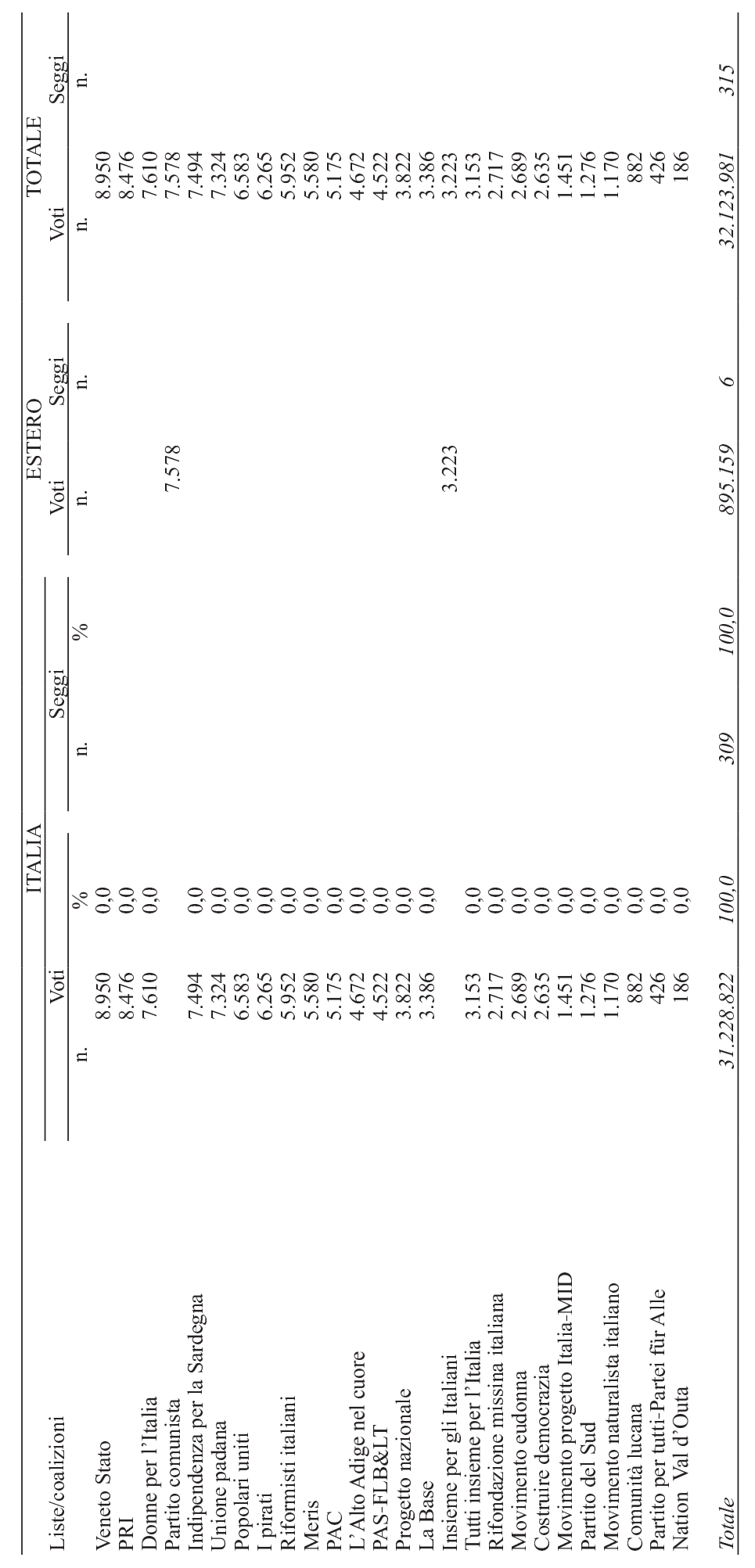

